\title{
The Isomorphism Problem On Classes of Automatic Structures
}

\author{
Dietrich Kuske ${ }^{1}$, Jiamou Liu ${ }^{2}$, and Markus Lohrey ${ }^{2, *}$ \\ 1 Centre national de la recherche scientifique (CNRS) and Laboratoire Bordelais de Recherche en \\ Informatique (LaBRI), Bordeaux, France \\ 2 Universität Leipzig, Institut für Informatik, Germany \\ kuske@labri.fr, liujiamou@gmail.com, lohrey@informatik.uni-leipzig.de
}

\begin{abstract}
Automatic structures are finitely presented structures where the universe and all relations can be recognized by finite automata. It is known that the isomorphism problem for automatic structures is complete for $\Sigma_{1}^{1}$; the first existential level of the analytical hierarchy. Several new results on isomorphism problems for automatic structures are shown in this paper: (i) The isomorphism problem for automatic equivalence relations is complete for $\Pi_{1}^{0}$ (first universal level of the arithmetical hierarchy). (ii) The isomorphism problem for automatic trees of height $n \geq 2$ is $\Pi_{2 n-3}^{0}$-complete. (iii) The isomorphism problem for automatic linear orders is not arithmetical. This solves some open questions of Khoussainov, Rubin, and Stephan.
\end{abstract}

\section{Introduction}

The idea of an automatic structure goes back to Büchi and Elgot who used finite automata to decide, e.g., Presburger arithmetic 5. Automaton decidable theories 8 and automatic groups 6 are similar concepts. A systematic study was initiated by Khoussainov and Nerode [13] who also coined the name "automatic structure". In essence, a structure is automatic if the elements of the universe can be represented as strings from a regular language and every relation of the structure can be recognized by a finite state automaton with several heads that proceed synchronously. Automatic structures received increasing interest over the last years [1311114]15]16 22]. One of the main motivations for investigating automatic structures is that their first-order theories can be decided uniformly (i.e., the input is an automatic presentation and a first-order sentence).

Automatic structures form a subclass of recursive (or computable) structures. A structure is recursive, if its domain as well as all relations are recursive sets of finite words (or naturals). A wellstudied problem for recursive structures is the isomorphism problem, where it is asked whether two given recursive structures over the same signature (encoded by Turing-machines for the domain and all relations) are isomorphic. It is well known that the isomorphism problem for recursive structures is complete for the first level of the analytical hierarchy $\Sigma_{1}^{1}$. In fact, $\Sigma_{1}^{1}$-completeness holds for many subclasses of recursive structures, e.g., for linear orders, trees, undirected graphs, Boolean algebras, Abelian p-groups, see 477. $\Sigma_{1}^{1}$-completeness of the isomorphism problem for a class of recursive structures implies non-existence of a good classification (in the sense of [4]) for that class 4 .

In [14], it was shown that also for automatic structures the isomorphism problem is $\Sigma_{1}^{1}$ complete. By a direct interpretation, it follows that for the following classes the isomorphism problem is still $\Sigma_{1}^{1}$-complete [18]: automatic successor trees, automatic undirected graphs, automatic commutative monoids, automatic partial orders, automatic lattices of height 4, and automatic 1-ary functions. On the other hand, the isomorphism problem is decidable for automatic ordinals [15] and automatic Boolean algebras [14]. An intermediate class is the class of all locallyfinite automatic graphs, for which the isomorphism problem is complete for $\Pi_{3}^{0}$ (third level of the arithmetical hierarchy) [21].

\footnotetext{
* The second and third author are supported by the DFG research project GELO.

${ }^{1}$ For background on the arithmetical hierarchy see, e.g., [19].
} 
For many interesting classes of automatic structures, the exact status of the isomorphism problem is open. In the recent survey [22] it was asked for instance, whether the isomorphism problem is decidable for automatic equivalence relations and automatic linear orders. For the latter class, this question was already asked in [15]. In this paper, we answer these questions. Our main results are:

- The isomorphism problem for automatic equivalence relations is $\Pi_{1}^{0}$-complete.

- The isomorphism problem for automatic successor trees of finite height $k \geq 2$ (where the height of a tree is the maximal number of edges along a maximal path) is $\Pi_{2 k-3}^{0}$-complete.

- The isomorphism problem for automatic linear orders is hard for every level of the arithmetical hierarchy.

Most hardness proofs for automatic structures, in particular the $\Sigma_{1}^{1}$-hardness proof for the isomorphism problem of automatic structures from [14, use transition graphs of Turing-machines (these graphs are easily seen to be automatic). This technique seems to fail for inherent reasons, when trying to prove our new results. The reason is most obvious for equivalence relations and linear orders. These structures are transitive but the transitive closure of the transition graph of a Turing-machine cannot be automatic in general (it's first-order theory is undecidable in general). Hence, we have to use a new strategy. Our proofs are based on the undecidability of Hilbert's $10^{\text {th }}$ problem. Recall that Matiyasevich proved that every recursively enumerable set of natural numbers is Diophantine [17. This fact was used by Honkala to show that it is undecidable whether the range of a rational power series is $\mathbb{N}[9$. Using a similar encoding, we show that the isomorphism problem for automatic equivalence relations is $\Pi_{1}^{0}$-complete. Next, we extend our technique in order to show that the isomorphism problem for automatic successor trees of height $k \geq 2$ is $\Pi_{2 k-3}^{0}$-complete. In some sense, our result for equivalence relations makes up the induction base $k=2$. Finally, using a similar but technically more involved reduction, we can show that the isomorphism problem for automatic linear orders is hard for every level of the arithmetical hierarchy. In fact, since our proof is uniform on the levels in the arithmetical hierarchy, it follows that the isomorphism problem for automatic linear orders is at least as hard as true arithmetic (the first-order theory of $(\mathbb{N} ;+, \times))$. At the moment it remains open whether the isomorphism problem for automatic linear orders is $\Sigma_{1}^{1}$-complete.

\section{Preliminaries}

Let $\mathbb{N}_{+}=\{1,2,3, \ldots\}$. Let $p\left(x_{1}, \ldots, x_{n}\right) \in \mathbb{N}\left[x_{1}, \ldots, x_{n}\right]$ be a polynomial with non-negative integer coefficients. We define

$$
\operatorname{Img}_{+}(p)=\left\{p\left(y_{1}, \ldots, y_{n}\right) \mid y_{1}, \ldots, y_{n} \in \mathbb{N}_{+}\right\} .
$$

If $p$ is not the zero-polynomial, then $\operatorname{Img}_{+}(p) \subseteq \mathbb{N}_{+}$.

Details on the arithmetical hierarchy can be found for instance in [19]. With $\Sigma_{n}^{0}$ we denote the $n^{\text {th }}$ (existential) level of the arithmetical hierarchy; it is the class of all subsets $A \subseteq \mathbb{N}$ such that there exists a recursive predicate $P \subseteq \mathbb{N}^{n+1}$ with

$$
A=\left\{a \in \mathbb{N} \mid \exists x_{1} \forall x_{2} \cdots Q x_{n}:\left(a, x_{1}, \ldots, x_{n}\right) \in P\right\},
$$

where $Q=\exists(Q=\forall)$ for $n$ odd (even). The set of complements of $\Sigma_{n}^{0}$-sets is denoted by $\Pi_{n}^{0}$. By fixing some effective encoding of strings by natural numbers, we can talk about $\Sigma_{n}^{0}$-sets and $\Pi_{n}^{0}$-sets of strings over an arbitrary alphabet. A typical example of a set, which does not belong to the arithmetical hierarchy is true arithmetic, i.e., the first-order theory of $(\mathbb{N} ;+, \times)$, which we denote by $\operatorname{FOTh}(\mathbb{N} ;+, \times)$.

We assume basic terminologies and notations in automata theory (see, for example, [10]). For a fixed alphabet $\Sigma$, a non-deterministic finite automaton is a tuple $\mathcal{A}=(S, \Delta, I, F)$ where $S$ is the set of states, $\Delta \subseteq S \times \Sigma \times S$ is the transition relation, $I \subseteq S$ is a set of initial states, and $F \subseteq S$ is the set of accepting states. A run of $\mathcal{A}$ on a word $u=a_{1} a_{2} \cdots a_{n}\left(a_{1}, a_{2} \ldots, a_{n} \in \Sigma\right)$ is 
a word over $\Delta$ of the form $r=\left(q_{0}, a_{1}, q_{1}\right)\left(q_{1}, a_{2}, q_{2}\right) \cdots\left(q_{n-1}, a_{n}, q_{n}\right)$, where $q_{0} \in I$. If moreover $q_{n} \in F$, then $r$ is an accepting run of $\mathcal{A}$ on $u$. We will only apply these definitions in case $n>0$, i.e., we will only speak of (accepting) runs on non-empty words.

Given two automata $\mathcal{A}_{1}=\left(S_{1}, \Delta_{1}, I_{1}, F_{1}\right)$ and $\mathcal{A}_{2}=\left(S_{1}, \Delta_{2}, I_{1}, F_{1}\right)$ over the same alphabet $\Sigma$, we use $\mathcal{A}_{1} \uplus \mathcal{A}_{2}$ to denote the automaton obtained by taking the disjoint union of $\mathcal{A}_{1}$ and $\mathcal{A}_{2}$. Note that for any word $u \in \Sigma^{+}$, the number of accepting runs of $\mathcal{A}_{1} \uplus \mathcal{A}_{2}$ on $u$ is equal to the sum of the numbers of accepting runs of $\mathcal{A}_{1}$ and $\mathcal{A}_{2}$ on $u$. We use $\mathcal{A}_{1} \times \mathcal{A}_{2}$ to denote the Cartesian product of $\mathcal{A}_{1}$ and $\mathcal{A}_{2}$. It is the automaton $\left(S_{1} \times S_{2}, \Delta, I_{1} \times I_{2}, F_{1} \times F_{2}\right)$, where

$$
\Delta=\left\{\left(\left(p_{1}, p_{2}\right), \sigma,\left(q_{1}, q_{2}\right)\right) \mid\left(p_{1}, \sigma, q_{1}\right) \in \Delta_{1},\left(p_{2}, \sigma, q_{2}\right) \in \Delta_{2}\right\} .
$$

Then, clearly, the number of accepting runs of $\mathcal{A}_{1} \times \mathcal{A}_{2}$ on a word $u \in L\left(A_{1}\right) \cap L\left(A_{2}\right)$ is the product of the numbers of accepting runs of $\mathcal{A}_{1}$ and $\mathcal{A}_{2}$ on $u$. In particular, if $\mathcal{A}_{1}$ is deterministic, then the number of accepting runs of $\mathcal{A}_{1} \times \mathcal{A}_{2}$ on $u \in L\left(\mathcal{A}_{1}\right) \cap L\left(A_{2}\right)$ is the same as the number of accepting runs of $\mathcal{A}_{2}$ on $u$. In the following, if $\mathcal{A}$ is a non-deterministic automaton and $D$ is a regular language, we write $D \uplus \mathcal{A}\left(\right.$ resp. $D \cap \mathcal{A}$ ) for the automaton $\mathcal{A}_{D} \uplus \mathcal{A}$ (resp. $\mathcal{A}_{D} \times \mathcal{A}$ ), where $\mathcal{A}_{D}$ is some deterministic automaton for the language $D$.

We use synchronous n-tape automata to recognize $n$-ary relations. Such automata have $n$ input tapes, each of which contains one of the input words. The $n$ tapes are read in parallel until all input words are processed. Formally, let $\Sigma_{\diamond}=\Sigma \cup\{\diamond\}$ where $\diamond \notin \Sigma$. For words $w_{1}, w_{2}, \ldots, w_{n} \in \Sigma^{*}$, their convolution is a word $w_{1} \otimes \cdots \otimes w_{n} \in\left(\Sigma_{\diamond}^{n}\right)^{*}$ with length $\max \left\{\left|w_{1}\right|, \ldots,\left|w_{n}\right|\right\}$, and the $k^{\text {th }}$ symbol of $w_{1} \otimes \cdots \otimes w_{n}$ is $\left(\sigma_{1}, \ldots, \sigma_{n}\right)$ where $\sigma_{i}$ is the $k^{t h}$ symbol of $w_{i}$ if $k \leq\left|w_{i}\right|$, and $\sigma_{i}=\diamond$ otherwise. An $n$-ary relation $R$ is $F A$ recognizable if the set of all convolutions of tuples $\left(w_{1}, \ldots, w_{n}\right) \in R$ is a regular language.

A relational structure $\mathcal{S}$ consists of a domain $D$ and atomic relations on the set $D$. We will only consider structures with countable domain. If $\mathcal{S}_{1}$ and $\mathcal{S}_{2}$ are two structures over the same signature and with disjoint domains, then we write $\mathcal{S}_{1} \uplus \mathcal{S}_{2}$ for the union of the two structures. Hence, when writing $\mathcal{S}_{1} \uplus \mathcal{S}_{2}$, we implicitly express that the domains of $\mathcal{S}_{1}$ and $\mathcal{S}_{2}$ are disjoint. More generally, if $\left\{\mathcal{S}_{i} \mid i \in I\right\}$ is a class of pairwise disjoint structures over the same signature, then we denote with $\uplus\left\{\mathcal{S}_{i} \mid i \in I\right\}$ the union of these structures. A structure $\mathcal{S}$ is called automatic over $\Sigma$ if its domain is a regular subset of $\Sigma^{*}$ and each of its atomic relations is FA recognizable; any tuple $\mathbb{P}$ of automata that accept the domain and the relations of $\mathcal{S}$ is called an automatic presentation of $\mathcal{S}$; in this case, we write $\mathcal{S}(\mathbb{P})$ for $\mathcal{S}$. If an automatic structure $\mathcal{S}$ is isomorphic to a structure $\mathcal{S}^{\prime}$, then $\mathcal{S}$ is called an automatic copy of $\mathcal{S}^{\prime}$ and $\mathcal{S}^{\prime}$ is automatically presentable. In this paper we sometimes abuse the terminology referring to $\mathcal{S}^{\prime}$ as simply automatic and calling an automatic presentation of $\mathcal{S}$ also automatic presentation of $\mathcal{S}^{\prime}$. We also simplify our statements by saying "given/compute an automatic structure $\mathcal{S}$ " for "given/compute an automatic presentation $\mathbb{P}$ of a structure $\mathcal{S}(\mathbb{P})$ ". The structures $(\mathbb{N} ; \leq,+)$ and $(\mathbb{Q} ; \leq)$ are both automatic structures. On the other hand, $(\mathbb{N} ; \times)$ and $(\mathbb{Q} ;+)$ have no automatic copies (see 1222 and $[24$ ).

Consider FO $+\exists^{\infty}+\exists^{n, m}$, the first-order logic extended by the quantifiers $\exists^{\infty}$ (there exist infinitely many) and $\exists^{n, m} x$ (there exist finitely many and the exact number is congruent $n$ modulo $m$, where $m, n \in \mathbb{N}$ ). The following theorem from [2]8]13]21] lays out the main motivation for investigating automatic structures.

Theorem 1. From an automatic presentation $\mathbb{P}$ and a formula $\varphi(\bar{x}) \in \mathrm{FO}+\exists^{\infty}+\exists^{n, m}$ in the signature of $\mathcal{S}(\mathbb{P})$, one can compute an automaton whose language consists of those tuples $\bar{a}$ from $\mathcal{S}(\mathbb{P})$ that make $\varphi$ true. In particular, the $\mathrm{FO}+\exists^{\infty}+\exists^{n, m}$ theory of any automatic structure $\mathcal{S}$ is (uniformly) decidable.

Let $\mathcal{K}$ be a class of automatic structures closed under isomorphism. The isomorphism problem for $\mathcal{K}$ is the set of pairs $\left(\mathbb{P}_{1}, \mathbb{P}_{2}\right)$ of automatic presentations with $\mathcal{S}\left(\mathbb{P}_{1}\right) \cong \mathcal{S}\left(\mathbb{P}_{2}\right) \in \mathcal{K}$. The isomorphism problem for the class of all automatic structures is complete for $\Sigma_{1}^{1}$ - the first level of the analytical hierarchy [14] (this holds already for automatic successor trees). However, if one restricts to special subclasses of automatic structures, this complexity bound can be reduced. For example, for the class of automatic ordinals and also the class of automatic Boolean algebras, the isomorphism 
problem is decidable. Another interesting result is that the isomorphism problem for locally finite automatic graphs is $\Pi_{3}^{0}$-complete [21]. All these classes of automatic structures have the nice property that one can decide whether a given automatic presentation describes a structure from this class. Theorem 1 implies that this property also holds for the classes of equivalence relations, trees of height at most $k$, and linear orders, i.e., the classes considered in this paper.

\section{Automatic Equivalence Structures}

An equivalence structure is of the form $\mathcal{E}=(D ; E)$ where $E$ is an equivalence relation on $D$. In this section, we prove that the isomorphism problem for automatic equivalence structures is $\Pi_{1}^{0}$-complete. This result can be also deduced from our result for automatic trees (Section 4). But the case of equivalence structures is a good starting point for introducing our techniques.

Let $\mathcal{E}$ be an automatic equivalence structure. Define the function $h_{\mathcal{E}}: \mathbb{N} \cup\left\{\aleph_{0}\right\} \rightarrow \mathbb{N} \cup\left\{\aleph_{0}\right\}$ such that for all $n \in \mathbb{N} \cup\left\{\aleph_{0}\right\}, h_{\mathcal{E}}(n)$ equals the number of equivalence classes (possibly infinite) in $\mathcal{E}$ of size $n$. Note that for given $n \in \mathbb{N} \cup\left\{\aleph_{0}\right\}$, the value $h_{\mathcal{E}}(n)$ can be computed effectively: one can define in $\mathrm{FO}+\exists^{\infty}$ the set of all $\leq_{\text {llex-least element: } 2}$ that belong to an equivalence class of size $n$.

Given two automatic equivalence structures $\mathcal{E}_{1}=\left(D_{1} ; E_{1}\right)$ and $\mathcal{E}_{2}=\left(D_{2} ; E_{2}\right)$, deciding if $\mathcal{E}_{1} \cong \mathcal{E}_{2}$ amounts to checking if $h_{\mathcal{E}_{1}}=h_{\mathcal{E}_{2}}$. Therefore, the isomorphism problem for automatic equivalence structures is in $\Pi_{1}^{0}$.

For the $\Pi_{1}^{0}$ lower bound, we use a reduction from Hilbert's $10^{\text {th }}$ problem: Given a polynomial $p\left(x_{1}, \ldots, x_{k}\right) \in \mathbb{Z}\left[x_{1}, \ldots, x_{k}\right]$, decide whether the equation $p\left(x_{1}, \ldots, x_{k}\right)=0$ has a solution in $\mathbb{N}_{+}$ (for technical reasons, it is useful to exclude 0 in solutions). This problem is well-known to be undecidable, see e.g. [17. In fact, Matiyasevich constructed from a given (index of a) recursively enumerable set $X \subseteq \mathbb{N}_{+}$a polynomial $p\left(x_{1}, \ldots, x_{k}\right) \in \mathbb{Z}\left[x_{1}, \ldots, x_{k}\right]$ such that for all $n \in \mathbb{N}_{+}: n \in X$ if and only if $\exists y_{2}, \ldots, y_{k} \in \mathbb{N}_{+}: p\left(n, y_{2}, \ldots, y_{k}\right)=0$. Hence, the following set is $\Pi_{1}^{0}$-complete:

$$
\left\{\left(p_{1}(\bar{x}), p_{2}(\bar{x})\right) \in \mathbb{N}\left[x_{1}, \ldots, x_{k}\right]^{2} \mid \forall \bar{c} \in \mathbb{N}_{+}^{k}: p_{1}(\bar{c}) \neq p_{2}(\bar{c})\right\} .
$$

For a symbol $a$, let $\Sigma_{k}^{a}$ denote the alphabet

$$
\Sigma_{k}^{a}=\{a, \diamond\}^{k} \backslash\{(\diamond, \ldots, \diamond)\}
$$

and let $\sigma_{i}$ denote the $i^{t h}$ component of $\sigma \in \Sigma_{k}^{a}$. For $\bar{e}=\left(e_{1}, \ldots, e_{k}\right) \in \mathbb{N}_{+}^{k}$, write $a^{\bar{e}}$ for the word

$$
a^{e_{1}} \otimes a^{e_{2}} \otimes \cdots \otimes a^{e_{k}}
$$

For a language $L$, we write $\otimes_{k}(L)$ for the language

$$
\left\{u_{1} \otimes u_{2} \otimes \cdots \otimes u_{k} \mid u_{1}, \ldots, u_{k} \in L\right\} .
$$

Lemma 2. There exists an algorithm that, given a non-zero polynomial $p(\bar{x}) \in \mathbb{N}[\bar{x}]$ in $k$ variables, constructs a non-deterministic automaton $\mathcal{A}[p(\bar{x})]$ on the alphabet $\Sigma_{k}^{a}$ with $L(\mathcal{A}[p(\bar{x})])=\otimes_{k}\left(a^{+}\right)$ such that for all $\bar{c} \in \mathbb{N}_{+}^{k}: \mathcal{A}[p(\bar{x})]$ has exactly $p(\bar{c})$ accepting runs on input $a^{\bar{c}}$.

Proof. The automaton $\mathcal{A}[p(\bar{x})]$ is build by induction on the construction of the polynomial $p$, the base case is provided by the polynomials 1 and $x_{i}$.

Let $\mathcal{A}[1]$ be a deterministic automaton accepting $\otimes_{k}\left(a^{+}\right)$. Next, suppose $p\left(x_{1}, \ldots, x_{k}\right)=x_{i}$ for some $i \in\{1, \ldots, k\}$. Let $S=\left\{q_{1}, q_{2}\right\}, I=\left\{q_{1}\right\}$ and $F=\left\{q_{2}\right\}$. Define $\Delta$ as

$$
\Delta=\left\{\left(q_{1}, \sigma, q_{j}\right) \mid j \in\{1,2\}, \sigma \in \Sigma_{k}^{a}, \sigma_{i}=a\right\} \cup\left\{\left(q_{2}, \sigma, q_{2}\right) \mid \sigma \in \Sigma_{k}^{a}\right\} .
$$

When the automaton $\mathcal{A}[p(\bar{x})]=(S, I, \Delta, F)$ runs on an input word $a^{\bar{c}}$, it has exactly $c_{i}$ many times the chance to move from state $q_{1}$ to the final state $q_{2}$. Therefore there are exactly $c_{i}=p(\bar{c})$ many accepting runs on $a^{\bar{c}}$.

\footnotetext{
${ }^{2} \leq_{\text {llex }}$ denotes the length-lexicographical order on words.
} 
Let $p_{1}(\bar{x})$ and $p_{2}(\bar{x})$ be polynomials in $\mathbb{N}[\bar{x}]$. Assume as inductive hypothesis that there are two automata $\mathcal{A}\left[p_{1}(\bar{x})\right]$ and $\mathcal{A}\left[p_{2}(\bar{x})\right]$ such that for $i \in\{1,2\}$ the number of accepting runs of $\mathcal{A}\left[p_{i}(\bar{x})\right]$ on $a^{\bar{c}}$ equals $p_{i}(\bar{c})$.

For $p(\bar{x})=p_{1}(\bar{x})+p_{2}(\bar{x})$, set $\mathcal{A}[p(\bar{x})]=\mathcal{A}\left[p_{1}(\bar{x})\right] \uplus \mathcal{A}\left[p_{2}(\bar{x})\right]$. Then, the number of accepting runs of $\mathcal{A}[p(\bar{x})]$ on $a^{\bar{c}}$ is $p_{1}(\bar{c})+p_{2}(\bar{c})$.

For $p(\bar{x})=p_{1}(\bar{x}) \cdot p_{2}(\bar{x})$, let $\mathcal{A}[p(\bar{x})]=\mathcal{A}\left[p_{1}(\bar{x})\right] \times \mathcal{A}\left[p_{2}(\bar{x})\right]$. Then, the number of accepting runs of $\mathcal{A}[p(\bar{x})]$ on $a^{\bar{c}}$ is $p_{1}(\bar{c}) \cdot p_{2}(\bar{c})$.

Let $\mathcal{A}=(S, I, \Delta, F)$ be a non-deterministic finite automaton with alphabet $\Sigma$. We define an automaton $\operatorname{Run}_{\mathcal{A}}=\left(S, I, \Delta^{\prime}, F\right)$ with alphabet $\Delta$ and

$$
\Delta^{\prime}=\{(p,(p, a, q), q) \mid(p, a, q) \in \Delta\} .
$$

Let $\pi: \Delta^{*} \rightarrow \Sigma^{*}$ be the projection morphism with $\pi(p, a, q)=a$. The following lemma is immediate from the definition.

Lemma 3. For $u \in \Delta^{+}$we have: $u \in L\left(\operatorname{Run}_{\mathcal{A}}\right)$ if and only if $u$ forms an accepting run of $\mathcal{A}$ on $\pi(u)$ (which in particular implies $\pi(u) \in L(\mathcal{A})$ ).

This lemma implies that for all words $w \in \Sigma^{+},\left|\pi^{-1}(w) \cap L\left(\operatorname{Run}_{\mathcal{A}}\right)\right|$ equals the number of accepting runs of $\mathcal{A}$ on $w$. Note that this does not hold for $w=\varepsilon$.

Consider a non-zero polynomial $p(\bar{x}) \in \mathbb{N}\left[x_{1}, \ldots, x_{k}\right]$. Let the automaton $\mathcal{A}=\mathcal{A}[p(\bar{x})]$ satisfy the properties guaranteed by Lemma 2 and let $\operatorname{Run}_{\mathcal{A}}$ be as defined above. Define an automatic equivalence structure $\mathcal{E}(p)$ whose domain is $L\left(\operatorname{Run}_{\mathcal{A}}\right) \backslash\{\varepsilon\}$. Moreover, two words $u, v \in L\left(\operatorname{Run}_{\mathcal{A}}\right) \backslash$ $\{\varepsilon\}$ are equivalent if and only if $\pi(u)=\pi(v)$. By definition and Lemma 2, a natural number $y \in \mathbb{N}_{+}$belongs to $\operatorname{Img}_{+}(p)$ if and only if there exists a word $u \in L(\mathcal{A})$ with precisely $y$ accepting runs, if and only if $\mathcal{E}(p)$ contains an equivalence class of size $y$.

It is well known that the function $C: \mathbb{N} \times \mathbb{N} \rightarrow \mathbb{N}$ with

$$
C(x, y)=(x+y)^{2}+3 x+y
$$

is injective $\left(C(x, y) / 2\right.$ defines a pairing function, see e.g. 9]). In the following, let $\mathcal{E}_{\text {Good }}$ denote the countably infinite equivalence structure with

$$
h_{\mathcal{E}_{\text {Good }}}(n)= \begin{cases}\infty & \text { if } n \in\left\{C(y, z) \mid y, z \in \mathbb{N}_{+}, y \neq z\right\} \\ 0 & \text { otherwise. }\end{cases}
$$

Proposition 4. The set of automatic presentations $\mathbb{P}$ with $\mathcal{S}(\mathbb{P}) \cong \mathcal{E}_{\text {Good }}$ is hard for $\Pi_{1}^{0}$.

Proof. For non-zero polynomials $p_{1}(\bar{x}), p_{2}(\bar{x}) \in \mathbb{N}\left[x_{1}, \ldots, x_{k}\right]$, define the following three (non-zero) polynomials from $\mathbb{N}\left[x_{1}, \ldots, x_{k}\right]$ (with $k \geq 2$ ):

$$
S_{1}(\bar{x})=C\left(p_{1}(\bar{x}), p_{2}(\bar{x})\right), \quad S_{2}(\bar{x})=C\left(x_{1}+x_{2}, x_{1}\right), \quad S_{3}(\bar{x})=C\left(x_{1}, x_{1}+x_{2}\right) .
$$

Let $\mathcal{E}\left(S_{1}\right), \mathcal{E}\left(S_{2}\right)$, and $\mathcal{E}\left(S_{3}\right)$ be the automatic equivalence structures corresponding to these polynomials according to the above definition. Finally, let $\mathcal{E}$ be the disjoint union of $\aleph_{0}$ many copies of these three equivalence structures.

If $p_{1}(\bar{c})=p_{2}(\bar{c})$ for some $\bar{c} \in \mathbb{N}_{+}^{k}$, then there is $y \in \mathbb{N}_{+}$such that $C(y, y) \in \operatorname{Img}_{+}\left(S_{1}\right)$. Therefore in $\mathcal{E}$ there is an equivalence class of size $C(y, y)$ and no such equivalence class exists in $\mathcal{E}_{\text {Good }}$. Hence $\mathcal{E} \not \mathcal{E}_{\text {Good. }}$.

Conversely, suppose that $p_{1}(\bar{c}) \neq p_{2}(\bar{c})$ for all $\bar{c} \in \mathbb{N}_{+}^{k}$. For all $y, z \in \mathbb{N}_{+}, \mathcal{E}$ contains an equivalence class of size $C(y, z)$ if and only if $C(y, z)$ belongs to $\operatorname{Img}_{+}\left(S_{1}\right) \cup \operatorname{Img}_{+}\left(S_{2}\right) \cup \operatorname{Img}_{+}\left(S_{3}\right)$, if and only if $y \neq z$, if and only if $\mathcal{E}_{\text {Good }}$ contains an equivalence class of size $C(y, z)$. Therefore, for any $s \in \mathbb{N}_{+}, \mathcal{E}$ contains an equivalence class of size $s$ if and only if $\mathcal{E}_{\text {Good }}$ contains an equivalence class of size $s$. Hence $\mathcal{E} \cong \mathcal{E}_{\text {Good }}$.

In summary, we have reduced the $\Pi_{1}^{0}$-hard problem

$$
\left\{\left(p_{1}(\bar{x}), p_{2}(\bar{x})\right) \in \mathbb{N}\left[x_{1}, \ldots, x_{k}\right]^{2} \mid k \geq 2, \forall \bar{c} \in \mathbb{N}_{+}^{k}: p_{1}(\bar{c}) \neq p_{2}(\bar{c})\right\}
$$

to the set of automatic presentations of $\mathcal{E}_{\text {Good }}$. Hence the proposition is proved. 
Theorem 5. The isomorphism problem for automatic equivalence structures is $\Pi_{1}^{0}$-complete.

Proof. At the beginning of this section, we already argued that the isomorphism problem is in $\Pi_{1}^{0}$; hardness follows immediately from Proposition 4 , since $\mathcal{E}_{\text {Good }}$ is necessarily automatic.

\section{Automatic Trees}

A tree is a structure $T=(V ; \leq)$, where $\leq$ is a partial order with a least element, called the root, and such that for every $x \in V$, the order $\leq$ restricted to the set $\{y \mid y \leq x\}$ of ancestors of $x$ is a finite linear order. The level of a node $x \in V$ is $|\{y \mid y<x\}| \in \mathbb{N}$. The height of $T$ is the supremum of the levels of all nodes in $V$; it may be infinite, but this paper deals with trees of finite height only. One may also view a tree as a directed graph $(V, E)$, where there is an edge $(u, v) \in E$ if and only if $u$ is the largest element in $\{x \mid x<v\}$. The edge relation $E$ is FO-definable in $(V ; \leq)$. In this paper, we assume the partial order definition for trees, but will quite often refer to them as graphs for convenience. We use $\mathcal{T}_{n}$ to denote the class of automatic trees with height at most $n$. Let $n$ be fixed. Then the tree order $\leq$ is FO-definable in $T$ and this holds even uniformly for all trees from $\mathcal{T}_{n}$. Moreover, it is decidable whether a given automatic graph belongs to $\mathcal{T}_{n}$ (since the class of trees of height $n$ can be axiomatized in first-order logic).

As a corollary to Proposition 4, we get immediately that the isomorphism problem for automatic trees of height at most 2 is undecidable:

Corollary 6. There exists an automatic tree $T_{\mathrm{Good}}$ of height 2 such that the set of automatic presentations $\mathbb{P}$ with $\mathcal{S}(\mathbb{P}) \cong T_{\text {Good }}$ is $\Pi_{1}^{0}$-hard. Hence, the isomorphism problem for the class $\mathcal{T}_{2}$ of automatic trees of height at most 2 is $\Pi_{1}^{0}$-hard.

Proof. Let $\mathcal{E}=(V ; \equiv)$ be an automatic equivalence structure. Now build the tree $T(\mathcal{E})$ as follows:

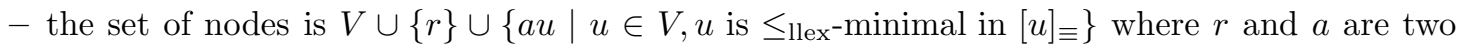
new letters

- $r$ is the root, its children are the words starting with $a$, and the children of $a u$ are the words from $[u] \equiv$.

Then it is clear that $T(\mathcal{E})$ is a tree of height at most 2 and that an automatic presentation for $T(\mathcal{E})$ can be computed from one for $\mathcal{E}$. Furthermore, $\mathcal{E} \cong \mathcal{E}_{\text {Good }}$ if and only if $T(\mathcal{E}) \cong T\left(\mathcal{E}_{\text {Good }}\right)$. Hence, indeed, the statement follows from Proposition 4

The hardness statement of Theorem 18 below is a generalization of this corollary to all the classes $\mathcal{T}_{n}$ for $n \geq 2$. But first, we prove an upper bound for the isomorphism problem for $\mathcal{T}_{n}$ :

Proposition 7. The isomorphism problem for the class $\mathcal{T}_{n}$ of automatic trees of height at most $n$ is

- decidable for $n=1$ and

- in $\Pi_{2 n-3}^{0}$ for all $n \geq 2$.

Proof. We first show that $T_{1} \cong T_{2}$ is decidable for automatic trees $T_{1}, T_{2} \in \mathcal{T}_{1}$ of height at most 1 : It suffices to compute the cardinality of $T_{i}(i \in\{1,2\})$ which is possible since the universes of $T_{1}$ and $T_{2}$ are regular languages.

Now let $n \geq 2$ and consider $T_{1}, T_{2} \in \mathcal{T}_{n}$. Let $T_{i}=\left(V_{i}, E_{i}\right)$, w.l.o.g. $V_{1} \cap V_{2}=\emptyset$, and $V=V_{1} \cup V_{2}$, $E=E_{1} \cup E_{2}$. For any node $u$ in $V$, let $T(u)$ denote the subtree (of either $T_{1}$ or $T_{2}$ ) rooted at $u$ and let $E(u)$ be the set of children of $u$. For $k=n-2, n-3, \ldots, 0$, we will define inductively a $\Pi_{2 n-2 k-3}^{0}$-predicate $\operatorname{iso}_{k}\left(u_{1}, u_{2}\right)$ for $u_{1}, u_{2} \in V$. This predicate expresses that $T\left(u_{1}\right) \cong T\left(u_{2}\right)$ provided $u_{1}$ and $u_{2}$ belong to level at least $k$. The result will follow since $T_{1} \cong T_{2}$ if and only if iso $_{0}\left(r_{1}, r_{2}\right)$ holds, where $r_{\sigma}$ is the root of $T_{\sigma}$. 
For $k=n-2$, the trees $T\left(u_{1}\right)$ and $T\left(u_{2}\right)$ have height at most 2 and we can define iso $\operatorname{son}_{n-2}\left(u_{1}, u_{2}\right)$ as follows:

$$
\forall \kappa \in \mathbb{N} \cup\left\{\aleph_{0}\right\} \forall \ell \geq 1\left(\begin{array}{r}
\exists x_{1}, \ldots, x_{\ell} \in E\left(u_{1}\right): \bigwedge_{1 \leq i<j \leq \ell} x_{i} \neq x_{j} \wedge \bigwedge_{i=1}^{\ell}\left|E\left(x_{i}\right)\right|=\kappa \\
\Longleftrightarrow y_{1}, \ldots, y_{\ell} \in E\left(u_{2}\right): \bigwedge_{1 \leq i<j \leq \ell} y_{i} \neq y_{j} \wedge \bigwedge_{i=1}^{\ell}\left|E\left(y_{i}\right)\right|=\kappa
\end{array}\right)
$$

In other words: for every $\kappa \in \mathbb{N} \cup\left\{\aleph_{0}\right\}, u_{1}$ and $u_{2}$ have the same number of children with exactly $\kappa$ children. Since FO $+\exists^{\infty}$ is uniformly decidable for automatic structures, this is indeed a $\Pi_{1}^{0}$ sentence (note that $2 n-2 k-3=1$ for $k=n-2$ ). For $0 \leq k<n-2$, we define $\operatorname{iso}_{k}\left(u_{1}, u_{2}\right)$ inductively as follows:

$$
\forall v \in E\left(u_{1}\right) \cup E\left(u_{2}\right) \forall \ell \geq 1\left(\begin{array}{r}
\exists x_{1}, \ldots, x_{\ell} \in E\left(u_{1}\right): \bigwedge_{1 \leq i<j \leq \ell} x_{i} \neq x_{j} \wedge \bigwedge_{i=1}^{\ell} \operatorname{iso}_{k+1}\left(v, x_{i}\right) \\
\Longleftrightarrow \exists y_{1}, \ldots, y_{\ell} \in E\left(u_{2}\right): \bigwedge_{1 \leq i<j \leq \ell} y_{i} \neq y_{j} \wedge \bigwedge_{i=1}^{\ell} \operatorname{iso}_{k+1}\left(v, y_{i}\right)
\end{array}\right)
$$

By quantifying over all $v \in E\left(u_{1}\right) \cup E\left(u_{2}\right)$, we quantify over all isomorphism types of trees that occur as a subtree rooted at a child of $u_{1}$ or $u_{2}$. For each of these isomorphism types $\tau$, we express that $u_{1}$ and $u_{2}$ have the same number of children $x$ with $T(x)$ of type $\tau$. Since by induction, iso $_{k+1}\left(v, x_{i}\right)$ and $\operatorname{iso}_{k+1}\left(v, y_{i}\right)$ are $\Pi_{2 n-2 k-5}^{0}$-statements, iso $k\left(u_{1}, u_{2}\right)$ is a $\Pi_{2 n-2 k-3}^{0}$-statement.

The rest of this section is devoted to proving that the isomorphism problem for the class $\mathcal{T}_{n}$ of automatic trees of height at most $n \geq 2$ is also $\Pi_{2 n-3}^{0}$-hard (and therefore complete). So let $P_{n}\left(x_{0}\right)$ be a $\Pi_{2 n-3}^{0}$-predicate. In the following lemma and its proof, all quantifiers with unspecified range run over $\mathbb{N}_{+}$.

Lemma 8. For $2 \leq i \leq n$, there are $\Pi_{2 i-3}^{0}$-predicates $P_{i}\left(x_{0}, x_{1}, y_{1}, x_{2}, y_{2}, \ldots, x_{n-i}, y_{n-i}\right)$ such that

(i) $P_{i+1}(\bar{x})$ is logically equivalent to $\forall x_{n-i} \exists y_{n-i}: P_{i}\left(\bar{x}, x_{n-i}, y_{n-i}\right)$ for $2 \leq i<n$ and

(ii) $\forall y_{n-i}: \neg P_{i}\left(\bar{x}, x_{n-i}, y_{n-i}\right)$ implies $\forall x_{n-i}^{\prime} \geq x_{n-i} \forall y_{n-i}: \neg P_{i}\left(\bar{x}, x_{n-i}^{\prime}, y_{n-i}\right)$,

where $\bar{x}=\left(x_{0}, x_{1}, y_{1}, \ldots, x_{n-i-1}, y_{n-i-1}\right)$.

Proof. The predicates $P_{i}$ are constructed by induction, starting with $i=n-1$ down to $i=2$ where the construction of $P_{i}$ does not assume that (i) or (ii) hold true for $P_{i+1}$.

So let $2 \leq i<n$ such that $P_{i+1}(\bar{x})$ is a $\Pi_{2(i+1)-3}^{0}$-predicate. Then there exists a $\Pi_{2 i-3}^{0}$-predicate $P\left(\bar{x}, x_{n-i}, y_{n-i}\right)$ such that $P_{i+1}(\bar{x})$ is logically equivalent to

$$
\forall x_{n-i} \exists y_{n-i}: P\left(\bar{x}, x_{n-i}, y_{n-i}\right) .
$$

But this is logically equivalent to

$$
\forall x_{n-i} \forall x_{n-i}^{\prime} \leq x_{n-i} \exists y_{n-i}: P\left(\bar{x}, x_{n-i}^{\prime}, y_{n-i}\right) .
$$

Let $\varphi\left(\bar{x}, x_{n-i}\right)$ be

$$
\forall x_{n-i}^{\prime} \leq x_{n-i} \exists y_{n-i}: P\left(\bar{x}, x_{n-i}^{\prime}, y_{n-i}\right)
$$

Then for any $x_{n-i} \in \mathbb{N}$,

$$
\neg \varphi\left(\bar{x}, x_{n-i}\right) \Longrightarrow \forall x \geq x_{n-i}: \neg \varphi(\bar{x}, x) \text {. }
$$

Since $\forall x_{n-i}^{\prime} \leq x_{n-i}$ is a bounded quantifier, the formula $\varphi\left(\bar{x}, x_{n-i}\right)$ belongs to $\Sigma_{2 i-2}^{0}$ (see for example [23, p. 61]). Thus there is a $\Pi_{2 i-3}^{0}$-predicate $P_{i}\left(\bar{x}, x_{n-i}, y_{n-i}\right)$ such that

$$
\varphi\left(\bar{x}, x_{n-i}\right) \Longleftrightarrow \exists y_{n-i}: P_{i}\left(\bar{x}, x_{n-i}, y_{n-i}\right) \text {. }
$$


Therefore (21) (and therefore $\left.P_{i+1}(\bar{x})\right)$ is logically equivalent to $\forall x_{n-i} \exists y_{n-i}: P_{i}\left(\bar{x}, x_{n-i}, y_{n-i}\right)$. Moreover,

$$
\begin{aligned}
\forall y_{n-i}: \neg P_{i}\left(\bar{x}, x_{n-i}, y_{n-i}\right) & \stackrel{(4)}{\Longrightarrow} \neg \varphi\left(\bar{x}, x_{n-i}\right) \\
& \stackrel{(3)}{\Longrightarrow} \forall x \geq x_{n-i}: \neg \varphi(\bar{x}, x) \\
& \stackrel{(4)}{\Longrightarrow} \forall x \geq x_{n-i} \forall y_{n-i}: \neg P_{i}\left(\bar{x}, x, y_{n-i}\right)
\end{aligned}
$$

This shows (ii).

Let us fix the predicates $P_{i}$ for the rest of Section 4 . By induction on $2 \leq i \leq n$, we will construct the following trees:

- test trees $T_{\bar{c}}^{i} \in \mathcal{T}_{i}$ for $\bar{c} \in \mathbb{N}_{+}^{1+2(n-i)}$ (which depend on $P_{i}$ ) and

- trees $U_{\kappa}^{i} \in \mathcal{T}_{i}$ for $\kappa \in \mathbb{N}_{+} \cup\{\omega\}$ (we assume the standard order on $\mathbb{N}_{+} \cup\{\omega\}$ ).

The idea is that $T_{\bar{c}}^{i} \cong U_{\kappa}^{i}$ if and only if $\kappa=1+\inf \left(\left\{x_{n-i} \mid \forall y_{n-i} \in \mathbb{N}_{+}: \neg P_{i}\left(\bar{c}, x_{n-i}, y_{n-i}\right)\right\} \cup\{\omega\}\right)$. We will not prove this equivalence, but the following simpler consequences for any $\bar{c} \in \mathbb{N}_{+}^{1+2(n-i)}$ :

(P1) $P_{i}(\bar{c})$ holds if and only if $T_{\bar{c}}^{i} \cong U_{\omega}^{i}$.

(P2) $P_{i}(\bar{c})$ does not hold if and only if $T_{\bar{c}}^{i} \cong U_{m}^{i}$ for some $m \in \mathbb{N}_{+}$.

The first property is certainly sufficient for proving $\Pi_{2 n-3}^{0}$-hardness (with $i=n$ ), the second property and therefore the trees $U_{m}^{i}$ for $m<\omega$ are used in the inductive step. We also need the following property for the construction.

(P3) No leaf of any of the trees $T_{\bar{c}}^{i}$ or $U_{\kappa}^{i}$ is a child of the root.

In the following section, we will describe the trees $T_{\bar{c}}^{i}$ and $U_{\kappa}^{i}$ of height at most $i$ and prove (P1) and (P2). Condition (P3) will be obvious from the construction. The subsequent section is then devoted to prove the effective automaticity of these trees.

\subsection{Construction of trees}

We start with a few definitions: A forest is a disjoint union of trees. Let $H_{1}$ and $H_{2}$ be two forests. The forest $H_{1}^{\omega}$ is the disjoint union of countably many copies of $H_{1}$. Formally, if $H_{1}=(V, E)$, then $H_{1}^{\omega}=\left(V \times \mathbb{N}, E^{\prime}\right)$ with $((v, i),(w, j)) \in E^{\prime}$ if and only if $(v, w) \in E$ and $i=j$. We write $H_{1} \sim H_{2}$ for $H_{1}^{\omega} \cong H_{2}^{\omega}$. Then $H_{1} \sim H_{2}$ if they are formed, up to isomorphism, by the same set of trees (i.e., any tree is isomorphic to some connected component of $H_{1}$ if and only if it is isomorphic to some connected component of $H_{2}$ ). If $H$ is a forest and $r$ does not belong to the domain of $H$, then we denote with $r \circ H$ the tree that results from adding $r$ to $H$ as new least element.

4.1.1 Induction base: construction of $\boldsymbol{T}_{\bar{c}}^{2}$ and $\boldsymbol{U}_{\boldsymbol{\kappa}}^{2}$ For notational simplicity, we write $k$ for $1+2(n-2)$. Hence, $P_{2}$ is a $k$-ary predicate. By Matiyasevich's theorem, we find two non-zero polynomials $p_{1}\left(x_{1}, \ldots, x_{\ell}\right), p_{2}\left(x_{1}, \ldots, x_{\ell}\right) \in \mathbb{N}[\bar{x}], \ell>k$, such that for any $\bar{c} \in \mathbb{N}_{+}^{k}$ :

$$
P_{2}(\bar{c}) \text { holds } \Longleftrightarrow \forall \bar{x} \in \mathbb{N}_{+}^{\ell-k}: p_{1}(\bar{c}, \bar{x}) \neq p_{2}(\bar{c}, \bar{x}) .
$$

For two numbers $m, n \in \mathbb{N}_{+}$, let $T[m, n]$ denote the tree of height 1 with exactly $C(m, n)$ leaves, where $C$ is the injective polynomial function from (11). Then define the following forests:

$$
\begin{aligned}
H^{2} & =\biguplus\left\{T[m, n] \mid m, n \in \mathbb{N}_{+}, m \neq n\right\} \\
H_{\bar{c}}^{2} & =H^{2} \uplus \biguplus\left\{T\left[p_{1}(\bar{c}, \bar{x})+x_{\ell+1}, p_{2}(\bar{c}, \bar{x})+x_{\ell+1}\right] \mid \bar{x} \in \mathbb{N}_{+}^{\ell-k}, x_{\ell+1} \in \mathbb{N}_{+}\right\} \\
J_{\kappa}^{2} & =H^{2} \uplus \biguplus\left\{T[x, x] \mid x \in \mathbb{N}_{+}, x>\kappa\right\} \quad \text { for } \kappa \in \mathbb{N}_{+} \cup\{\omega\}
\end{aligned}
$$



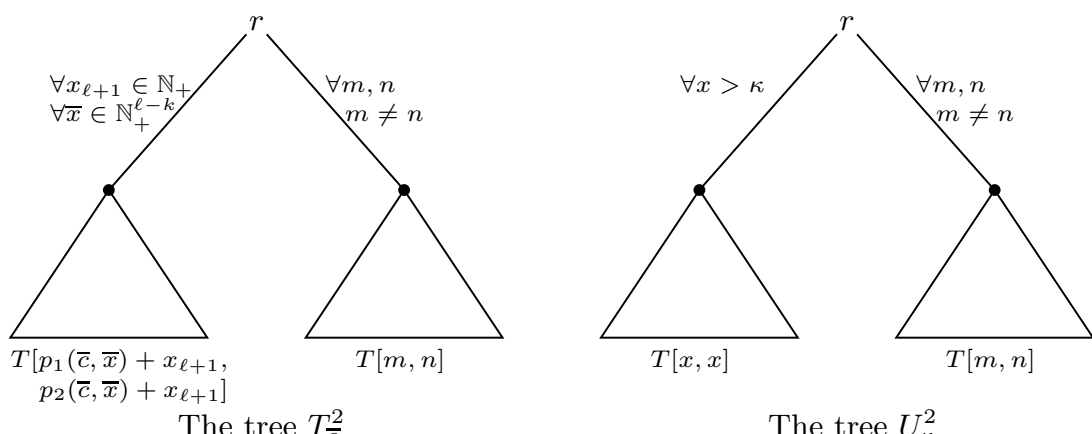

Fig. 1. The tree $T_{\bar{c}}^{2}$ and $U_{\kappa}^{2}$

The tree $U_{\kappa}^{2}$

Note that $J_{\omega}^{2}=H^{2}$. Moreover, the forests $J_{\kappa}^{2}\left(\kappa \in \mathbb{N}_{+} \cup\{\omega\}\right)$ are pairwise non-isomorphic, since $C$ is injective.

The trees $T_{\bar{c}}^{2}$ and $U_{\kappa}^{2}$, resp., are obtained from $H_{\bar{c}}^{2}$ and $J_{\kappa}^{2}$, resp., by taking countably many copies and adding a root:

$$
T_{\bar{c}}^{2}=r \circ\left(H_{\bar{c}}^{2}\right)^{\omega} \quad U_{\kappa}^{2}=r \circ\left(J_{\kappa}^{2}\right)^{\omega},
$$

see Figure 1.

The following lemma (stating (P1) for the $\Pi_{1}^{0}$-predicate $P_{2}$, i.e., for $i=2$ ) can be proved in a similar way as Theorem 5 .

Lemma 9. For any $\bar{c} \in \mathbb{N}_{+}^{k}$, we have:

$$
P_{2}(\bar{c}) \text { holds } \Longleftrightarrow H_{\bar{c}}^{2} \sim J_{\omega}^{2} \Longleftrightarrow T_{\bar{c}}^{2} \cong U_{\omega}^{2} .
$$

Proof. By (5), it suffices to show the first equivalence. So first assume $P_{2}(\bar{c})$ holds. We have to prove that the forests $H_{\bar{c}}^{2}$ and $J_{\omega}^{2}=H^{2}$ contain the same trees (up to isomorphism). Clearly, every tree from $H^{2}$ is contained in $H_{\bar{c}}^{2}$. For the other direction, let $\bar{x} \in \mathbb{N}_{+}^{\ell-k}$ and $x_{\ell+1} \in \mathbb{N}_{+}$. Then the tree $T\left[p_{1}(\bar{c}, \bar{x})+x_{\ell+1}, p_{2}(\bar{c}, \bar{x})+x_{\ell+1}\right]$ occurs in $H_{\bar{c}}^{2}$. Since $P_{2}(\bar{c})$ holds, we have $p_{1}(\bar{c}, \bar{x}) \neq p_{2}(\bar{c}, \bar{x})$ and therefore $p_{1}(\bar{c}, \bar{x})+x_{\ell+1} \neq p_{2}(\bar{c}, \bar{x})+x_{\ell+1}$. Hence this tree also occurs in $H^{2}$.

Conversely suppose $H_{\bar{c}}^{2} \sim H^{2}$ and let $\bar{x} \in \mathbb{N}_{+}^{\ell-k}$. Then the tree $T\left[p_{1}(\bar{c}, \bar{x})+1, p_{2}(\bar{c}, \bar{x})+1\right]$ occurs in $H_{\bar{c}}^{2}$ and therefore in $H^{2}$. Hence $p_{1}(\bar{c}, \bar{x}) \neq p_{2}(\bar{c}, \bar{x})$. Since $\bar{x}$ was chosen arbitrarily, this implies $P_{2}(\bar{c})$.

Now consider the forest $H_{\bar{c}}^{2}$ once more. If it contains a tree of the form $T[m, m]$ for some $m$ (necessarily $m \geq 2$ ), then it contains all trees $T[x, x]$ for $x \geq m$. Hence, $H_{\bar{c}}^{2} \sim J_{\kappa}^{2}$ for some $\kappa \in \mathbb{N}_{+} \cup\{\omega\}$, which implies $T_{\bar{c}}^{2} \cong U_{\kappa}^{2}$ for some $\kappa \in \mathbb{N}_{+} \cup\{\omega\}$. Thus, with Lemma 9 we get:

$$
P_{2}(\bar{c}) \text { does not hold } \Longleftrightarrow T_{\bar{c}}^{2} \not U_{\omega}^{2} \Longleftrightarrow \exists m \in \mathbb{N}_{+}: T_{\bar{c}}^{2} \cong U_{m}^{2}
$$

Hence we proved the following lemma, which states (P2) for the $\Pi_{1}^{0}$-predicate $P_{2}$, i.e., for $i=2$.

Lemma 10. For any $\bar{c} \in \mathbb{N}_{+}^{k}$, we have:

$$
P_{2}(\bar{c}) \text { does not hold } \Longleftrightarrow \exists m \in \mathbb{N}_{+}: T_{\bar{c}}^{2} \cong U_{m}^{2} .
$$

This finishes the construction of the trees $T_{\bar{c}}^{2}$ and $U_{\kappa}^{2}$ for $\kappa \in \mathbb{N}_{+} \cup\{\omega\}$, and the verification of properties (P1) and (P2). Clearly, also (P3) holds for $T_{\bar{c}}^{2}$ and $U_{\kappa}^{2}$ (all maximal paths have length 2).

4.1.2 Induction step: construction of $\boldsymbol{T}_{\bar{c}}^{\boldsymbol{i}+\mathbf{1}}$ and $\boldsymbol{U}_{\boldsymbol{\kappa}}^{i+1}$ For notational simplicity, we write again $k$ for $1+2(n-i-1)$ such that $P_{i+1}$ is a $k$-ary predicate and $P_{i}$ a $(k+2)$-ary one.

We now apply the induction hypothesis. For any $\bar{c} \in \mathbb{N}_{+}^{k}, x, y \in \mathbb{N}_{+}, \kappa \in \mathbb{N}_{+} \cup\{\omega\}$ let $T_{\bar{c} x y}^{i}$ and $U_{\kappa}^{i}$ be trees of height at most $i$ such that: 


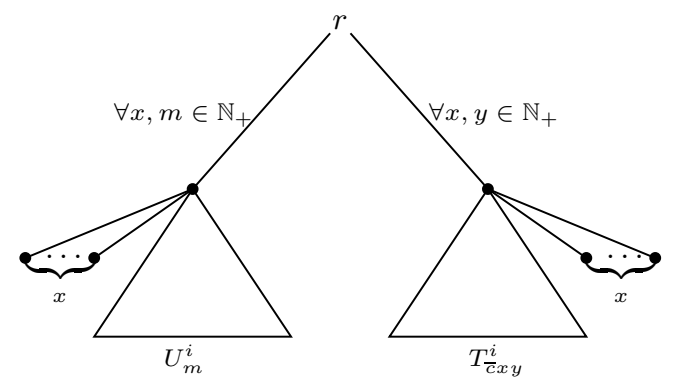

The tree $T_{\bar{c}}^{i+1}$

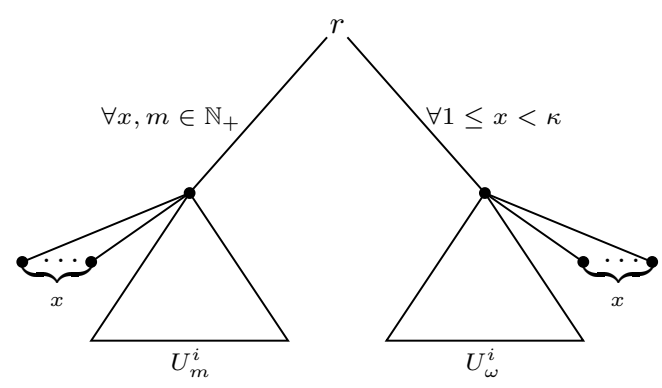

The tree $U_{\kappa}^{i+1}$

Fig. 2. The tree $T_{\bar{c}}^{i+1}$ and $U_{\kappa}^{i+1}$

- $P_{i}(\bar{c}, x, y)$ holds if and only if $T_{\bar{c} x y}^{i} \cong U_{\omega}^{i}$.

- $P_{i}(\bar{c}, x, y)$ does not hold if and only if $T_{\bar{c} x y}^{i} \cong U_{m}^{i}$ for some $m \in \mathbb{N}_{+}$.

In a first step, we build the trees $T_{\bar{c} x y}^{\prime}$ and $U_{\kappa, x}^{\prime}\left(x \in \mathbb{N}_{+}\right)$from $T_{\bar{c} x y}^{i}$ and $U_{\kappa}^{i}$, resp., by adding $x$ leaves as children of the root. This ensures

$$
\begin{aligned}
T_{\bar{c} x y}^{\prime} \cong T_{\bar{c} x^{\prime} y^{\prime}}^{\prime} & \Longleftrightarrow x=x^{\prime} \wedge T_{\bar{c} x y}^{i} \cong T_{\bar{c} x^{\prime} y^{\prime}}^{i} \text { and } \\
T_{\bar{c} x y}^{\prime} \cong U_{\kappa, x^{\prime}}^{\prime} & \Longleftrightarrow x=x^{\prime} \wedge T_{\bar{c} x y}^{i} \cong U_{\kappa}^{i},
\end{aligned}
$$

since, by property (P3), no leaf of any of the trees $T_{\bar{c} x y}^{i}$ or $U_{\kappa}^{i}$ is a child of the root. Next, we collect these trees into forests as follows:

$$
\begin{aligned}
& H^{i+1}=\biguplus\left\{U_{m, x}^{\prime} \mid x, m \in \mathbb{N}_{+}\right\}, \\
& H_{\bar{c}}^{i+1}=H^{i+1} \uplus \biguplus\left\{T_{\bar{c} x y}^{\prime} \mid x, y \in \mathbb{N}_{+}\right\}, \text {and } \\
& J_{\kappa}^{i+1}=H^{i+1} \uplus \biguplus\left\{U_{\omega, x}^{\prime} \mid 1 \leq x<\kappa\right\} \text { for } \kappa \in \mathbb{N}_{+} \cup\{\omega\} .
\end{aligned}
$$

The trees $T_{\bar{c}}^{i+1}$ and $U_{\kappa}^{i+1}$, resp., are then obtained from the forests $H_{\bar{c}}^{i+1}$ and $J_{\kappa}^{i+1}$, resp., by taking countably many copies and adding a root:

$$
T_{\bar{c}}^{i+1}=r \circ\left(H_{\bar{c}}^{i+1}\right)^{\omega} \quad \text { and } \quad U_{\kappa}^{i+1}=r \circ\left(J_{\kappa}^{i+1}\right)^{\omega},
$$

see Figure 2 ,

Note that the height of any of these trees is one more than the height of the forests defining them and therefore at most $i+1$. Since none of the connected components of the forests $H_{\bar{c}}^{i+1}$ and $J_{\kappa}^{i+1}$ is a singleton, none of the trees in (8) has a leaf that is a child of the root and therefore (P3) holds.

Lemma 11. For all $\bar{c} \in \mathbb{N}_{+}^{k}$ we have

$$
P_{i+1}(\bar{c}) \text { holds } \Longleftrightarrow H_{\bar{c}}^{i+1} \sim J_{\omega}^{i+1} \Longleftrightarrow T_{\bar{c}}^{i+1} \cong U_{\omega}^{i+1} .
$$

Proof. Again, we only have to prove the first equivalence.

First assume $H_{\bar{c}}^{i+1} \sim J_{\omega}^{i+1}$ and let $x \geq 1$ be arbitrary. We have to exhibit some $y \geq 1$ such that $P_{i}(\bar{c}, x, y)$ holds. Note that $U_{\omega, x}^{\prime}$ belongs to $J_{\omega}^{i+1}$ and therefore to $H_{\bar{c}}^{i+1}$. Since $U_{\omega, x}^{\prime} \neq U_{m, x^{\prime}}^{\prime}$ for any $m, x, x^{\prime} \in \mathbb{N}_{+}$, this implies the existence of $x^{\prime}, y^{\prime} \geq 1$ with $T_{\bar{c} x^{\prime} y^{\prime}}^{\prime} \cong U_{\omega, x}^{\prime}$. By (77), this is equivalent with $x=x^{\prime}$ and $T_{\bar{c} x y^{\prime}}^{i} \cong U_{\omega}^{i}$. Now the induction hypothesis implies that $P_{i}\left(\bar{c}, x, y^{\prime}\right)$ holds. Since $x \geq 1$ was chosen arbitrarily, we can deduce $P_{i+1}(\bar{c})$.

Conversely suppose $P_{i+1}(\bar{c})$. Let $T$ belong to $H_{\bar{c}}^{i+1}$. By the induction hypothesis, it is one of the trees $U_{\kappa, x}^{\prime}$ for some $x \in \mathbb{N}_{+}, \kappa \in \mathbb{N}_{+} \cup\{\omega\}$. In any case, it also belongs to $J_{\omega}^{i+1}$. Hence it remains to show that any tree of the form $U_{\omega, x}^{\prime}$ belongs to $H_{\bar{c}}^{i+1}$. So let $x \in \mathbb{N}_{+}$. Then, by $P_{i+1}(\bar{c})$, there exists $y \in \mathbb{N}_{+}$with $P_{i}(\bar{c}, x, y)$. By the induction hypothesis, we have $T_{\bar{c} x y}^{i} \cong U_{\omega}^{i}$ and therefore $T_{\bar{c} x y}^{\prime} \cong U_{\omega, x}^{\prime}$ (which belongs to $H_{\bar{c}}^{i+1}$ by the very definition). 
Lemma 12. For all $\bar{c} \in \mathbb{N}_{+}^{k}$ there exists $\kappa \in \mathbb{N}_{+} \cup\{\omega\}$ such that $T_{\bar{c}}^{i+1} \cong U_{\kappa}^{i+1}$.

Proof. It suffices to prove that $H_{\bar{c}}^{i+1} \sim J_{\kappa}^{i+1}$ for some $\kappa \in \mathbb{N}_{+} \cup\{\omega\}$. Choose $\kappa$ as the smallest value in $\mathbb{N}_{+} \cup\{\omega\}$ such that

$$
\forall x \geq \kappa \forall y: \neg P_{i}(\bar{c}, x, y)
$$

holds. By property (ii) from Lemma 8 for $P_{i}$, we get

$$
\forall 1 \leq x<\kappa \exists y: P_{i}(\bar{c}, x, y) .
$$

By the induction hypothesis, we get

$$
\forall x \geq \kappa \forall y: T_{\bar{c} x y}^{\prime} \neq U_{\omega, x}^{\prime} \text { and } \forall 1 \leq x<\kappa \exists y: T_{\bar{c} x y}^{\prime} \cong U_{\omega, x}^{\prime} .
$$

It follows that $H_{\bar{c}}^{i+1}$ contains, apart from the trees in $H^{i+1}=\biguplus\left\{U_{m, x}^{\prime} \mid x, m \in \mathbb{N}_{+}\right\}$, exactly the trees from $\biguplus\left\{U_{\omega, x}^{\prime} \mid 1 \leq x<\kappa\right\}$. Hence, $H_{\bar{c}}^{i+1} \sim J_{\kappa}^{i+1}$.

Lemma 11 and 12 immediately imply:

Lemma 13. For all $\bar{c} \in \mathbb{N}_{+}^{k}$ we have

$$
P_{i+1}(\bar{c}) \text { does not hold } \Longleftrightarrow \exists m \in \mathbb{N}_{+}: T_{\bar{c}}^{i+1} \cong U_{m}^{i+1} .
$$

In summary, we obtained the following:

Proposition 14. Let $n \geq 2$ and let $P(x)$ be a $\Pi_{2 n-3}^{0}$-predicate. Then, for any $c \in \mathbb{N}_{+}$, we have

$$
P(c) \text { holds } \Longleftrightarrow T_{c}^{n} \cong U_{\omega}^{n} .
$$

To infer the $\Pi_{2 n-3}^{0}$-hardness of the isomorphism problem for $\mathcal{T}_{n}$ from this proposition, it remains to be shown that the trees $T_{c}^{n}$ and $U_{\omega}^{n}$ are effectively automatic - this is the topic of the next section.

\subsection{Automaticity}

For constructing automatic presentations for the trees from the previous section, it is actually easier to work with dags (directed acyclic graphs). The height of a dag $D$ is the length (number of edges) of a longest directed path in $D$. We only consider dags of finite height. A root of a dag is a node without incoming edges. A dag $D=(V, E)$ can be unfolded into a forest unfold $(D)$ in the usual way: Nodes of unfold $(D)$ are directed paths in $D$ that cannot be extended to the left (i.e., the initial node of the path is a root) and there is an edge between a path $p$ and a path $p^{\prime}$ if and only if $p^{\prime}$ extends $p$ by one more node. For a node $v \in V$ of $D$, we define the tree $\operatorname{unfold}(D, v)$ as follows: First we restrict $D$ to those nodes that are reachable from $v$ and then we unfold the resulting dag. We need the following lemma.

Lemma 15. From given $k \in \mathbb{N}$ and an automatic dag $D=(V, E)$ of height at most $k$, one can construct effectively an automatic presentation $\mathbb{P}$ with $\mathcal{S}(\mathbb{P}) \cong \operatorname{unfold}(D)$.

Proof. The universe for our automatic copy of unfold $(D)$ is the set $P$ of all convolutions $v_{1} \otimes v_{2} \otimes$ $\cdots \otimes v_{m}$, where $v_{1}$ is a root and $\left(v_{i}, v_{i+1}\right) \in E$ for all $1 \leq i<m$. Since $D$ has height at most $k$, we have $m \leq k$. Since the edge relation of $D$ is automatic and since the set of all roots in $D$ is first-order definable and hence regular, $P$ is indeed a regular set. Moreover, the edge relation of unfold $(D)$ becomes clearly FA recognizable on $P$.

For $2 \leq i \leq n$, let us consider the following forest:

$$
F^{i}=\biguplus\left\{T_{\bar{c}}^{i} \mid \bar{c} \in \mathbb{N}_{+}^{1+2(n-i)}\right\} \uplus \biguplus\left\{U_{\kappa}^{i} \mid \kappa \in \mathbb{N}_{+} \cup\{\omega\}\right\} .
$$

Technically, this section proves by induction over $i$ the following statement: 
Proposition 16. For $2 \leq i \leq n$, there exists an automatic copy $\mathcal{F}^{i}$ of $F^{i}$ and an isomorphism $f^{i}: F^{i} \rightarrow \mathcal{F}^{i}$ that maps

1. the root of the tree $T_{\bar{c}}^{i}$ to $a^{\bar{c}}$ (for all $\bar{c} \in \mathbb{N}_{+}^{1+2(n-i)}$ ),

2. the root of the tree $U_{\omega}^{i}$ to $\varepsilon$, and

3. the root of the tree $U_{m}^{i}$ to $b^{m}$ (for all $m \in \mathbb{N}_{+}$).

This will give the desired result since $T_{c}^{n}$ is then isomorphic to the connected component of $\mathcal{F}^{n}$ that contains the word $a^{c}$ (and similarly for $U_{\kappa}^{n}$ ). Note that this connected component is automatic by Theorem 11 since the forest $\mathcal{F}^{n}$ has bounded height. Moreover, an automatic presentation for the connected component containing $a^{c}$ can be computed from $c$.

By Lemma 15, it suffices to construct an automatic dag $\mathcal{D}^{i}$ such that there is an isomorphism $h: \operatorname{unfold}\left(\mathcal{D}^{i}\right) \rightarrow \mathcal{F}^{i}$ that is the identity on the set of roots of $\mathcal{D}^{i}$.

4.2.1 Induction base: the automatic dag $\mathcal{D}^{2}$ Recall the definitions of $\Sigma_{\ell}^{a}$, $a^{\bar{e}}$, and $\otimes_{k}(L)$ from Section 3

Lemma 17. From $\ell \in \mathbb{N}_{+}, q_{1}, q_{2} \in \mathbb{N}\left[x_{1}, \ldots, x_{\ell}\right]$, and a symbol a, one can compute an automatic forest of height 1 over an alphabet $\Sigma_{\ell}^{a} \uplus \Gamma$ such that

- the roots are the words from $\otimes_{\ell}\left(a^{+}\right)$,

- the leaves are words from $\Gamma^{+}$, and

- the tree rooted at $a^{\bar{e}}$ is isomorphic to $T\left[q_{1}(\bar{e}), q_{2}(\bar{e})\right]$.

Proof. Set $p\left(x_{1}, \ldots, x_{\ell}\right)=C\left(q_{1}\left(x_{1}, \ldots, x_{\ell}\right), q_{2}\left(x_{1}, \ldots, x_{\ell}\right)\right)$ and recall the definition of the automata $\mathcal{A}[p]$ and $\operatorname{Run}_{\mathcal{A}[p]}$ from Section [3. Recall also that we let $\pi$ be the projection with $\pi(p, a, q)=a$ for a transition $(p, a, q)$ of $\mathcal{A}[p]$. Then let

$$
\begin{aligned}
L\left[q_{1}, q_{2}\right] & =\otimes_{\ell}\left(a^{+}\right) \cup\left(\pi^{-1}\left(\otimes_{\ell}\left(a^{+}\right)\right) \cap L\left(\operatorname{Run}_{\mathcal{A}[p]}\right)\right) \text { and } \\
E\left[q_{1}, q_{2}\right] & =\left\{(u, v) \mid u \in \otimes_{\ell}\left(a^{+}\right), v \in \pi^{-1}(u) \cap L\left(\operatorname{Run}_{\mathcal{A}[p]}\right)\right\} .
\end{aligned}
$$

Then $L\left[q_{1}, q_{2}\right]$ is regular and $E\left[q_{1}, q_{2}\right]$ is FA recognizable, i.e., the pair $\left(L\left[q_{1}, q_{2}\right] ; E\left[q_{1}, q_{2}\right]\right)$ is an automatic graph. It is actually a forest of height 1 , the words from $\otimes_{\ell}\left(a^{+}\right)$form the roots, and the tree rooted at $a^{\bar{e}}$ has precisely $p(\bar{e})$ leaves, i.e., it is isomorphic to $T\left[q_{1}(\bar{e}), q_{2}(\bar{e})\right]$.

From now on, we use the notations from Section 4.1.1. Using Lemma17, we can compute automatic forests $\mathcal{F}_{1}$ and $\mathcal{F}_{2}$ over alphabets $\Sigma_{\ell+1}^{a} \uplus \Gamma_{1}$ and $\Sigma_{2}^{b} \uplus \Gamma_{2}$, respectively, such that

(a) the roots of $\mathcal{F}_{1}$ are the words from $\otimes_{\ell+1}\left(a^{+}\right)$,

(b) the roots of $\mathcal{F}_{2}$ are the words from $\otimes_{2}\left(b^{+}\right)$,

(c) the leaves of $\mathcal{F}_{i}$ are words from $\Gamma_{i}^{+}(i \in\{1,2\})$,

(d) the tree rooted at $a^{\bar{e} e_{\ell+1}}$ is isomorphic to $T\left[p_{1}(\bar{e})+e_{\ell+1}, p_{2}(\bar{e})+e_{\ell+1}\right]$ for $\bar{e} \in \mathbb{N}_{+}^{\ell}, e_{\ell+1} \in \mathbb{N}_{+}$,

(e) the tree rooted at $b^{e_{1} e_{2}}$ is isomorphic to $T\left[e_{1}, e_{2}\right]$ for $e_{1}, e_{2} \in \mathbb{N}_{+}$.

We can assume that the alphabets $\Gamma_{1}, \Gamma_{2}, \Sigma_{\ell+1}^{a}$, and $\Sigma_{2}^{b}$ are mutually disjoint. Let $\mathcal{F}=\left(V_{\mathcal{F}}, E_{\mathcal{F}}\right)$ be the disjoint union of $\mathcal{F}_{1}$ and $\mathcal{F}_{2}$; it is effectively automatic.

The universe of the automatic dag $\mathcal{D}^{2}$ is the regular language

$$
\otimes_{k}\left(a^{+}\right) \cup b^{*} \cup\left(\$^{*} \otimes V_{\mathcal{F}}\right),
$$

where $\$$ is a new symbol. We have the following edges:

- For $u, v \in V_{\mathcal{F}}, \$^{m} \otimes u$ is connected to $\$^{n} \otimes v$ if and only if $m=n$ and $(u, v) \in E_{\mathcal{F}}$. This produces $\aleph_{0}$ many copies of $\mathcal{F}$.

$-a^{\bar{c}}$ is connected to any word from $\mathbb{S}^{*} \otimes\left(\left\{a^{\bar{c} \bar{x}} \mid \bar{x} \in \mathbb{N}_{+}^{\ell-k+1}\right\} \cup\left\{b^{e_{1} e_{2}} \mid e_{1} \neq e_{2}\right\}\right)$. By point (d) and (e) above, this means that the tree unfold $\left(\mathcal{D}^{2}, a^{\bar{c}}\right)$ has $\aleph_{0}$ many subtrees isomorphic to $T\left[p_{1}(\bar{c} \bar{x})+x_{\ell+1}, p_{2}(\bar{c} \bar{x})+x_{\ell+1}\right]$ for $\bar{x} \in \mathbb{N}_{+}^{\ell-k}, x_{\ell+1} \in \mathbb{N}_{+}$and $T\left[e_{1}, e_{2}\right]$ for $e_{1}, e_{2} \in \mathbb{N}_{+}, e_{1} \neq e_{2}$. Hence, unfold $\left(\mathcal{D}^{2}, a^{\bar{c}}\right) \cong T_{\bar{c}}^{2}$. 

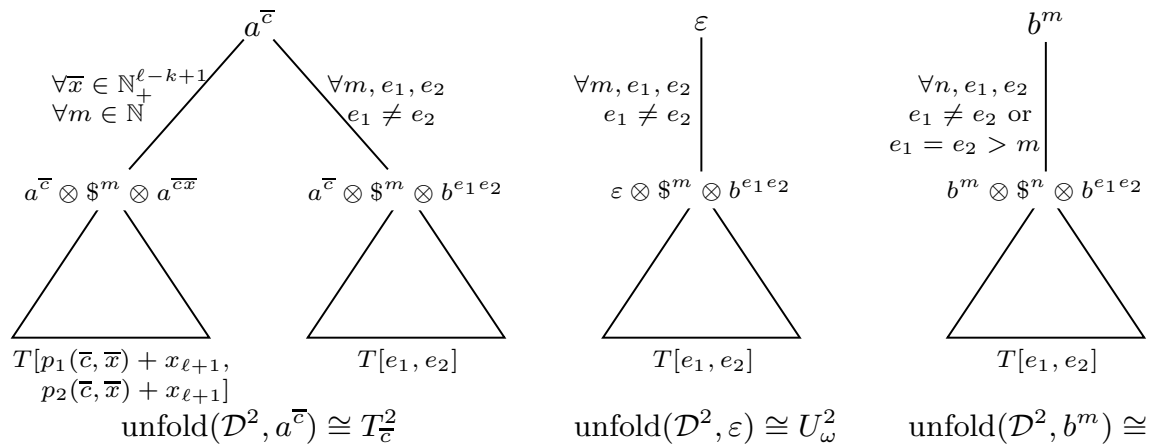

Fig. 3. Automatic presentation of $T_{\bar{c}}^{2}$ and $U_{\kappa}^{2}$

$-\varepsilon$ is connected to all words from $\$^{*} \otimes\left\{b^{e_{1} e_{2}} \mid e_{1} \neq e_{2}\right\}$. By (e) above, this means that the tree $\operatorname{unfold}\left(\mathcal{D}^{2}, \varepsilon\right)$ has $\aleph_{0}$ many subtrees isomorphic to $T\left[e_{1}, e_{2}\right]$ for $e_{1}, e_{2} \in \mathbb{N}_{+}, e_{1} \neq e_{2}$. Hence, $\operatorname{unfold}\left(\mathcal{D}^{2}, \varepsilon\right) \cong U_{\omega}^{2}$.

- $b^{m}\left(m \in \mathbb{N}_{+}\right)$is connected to all words from $\mathbb{S}^{*} \otimes\left\{b^{e_{1} e_{2}} \mid e_{1} \neq e_{2}\right.$ or $\left.e_{1}=e_{2}>m\right\}$. By (e) above, this means that the tree unfold $\left(\mathcal{D}^{2}, b^{m}\right)$ has $\aleph_{0}$ many subtrees isomorphic to $T\left[e_{1}, e_{2}\right]$ for all $e_{1}, e_{2} \in \mathbb{N}_{+}$with $e_{1} \neq e_{2}$ or $e_{1}=e_{2}>m$. Hence, $\operatorname{unfold}\left(\mathcal{D}^{2}, b^{m}\right) \cong U_{m}^{2}$.

Thus, unfold $\left(\mathcal{D}_{2}\right) \cong F^{2}$ and the roots are as required in Proposition 16, see Figure 3. Moreover, it is clear that $\mathcal{D}_{2}$ is automatic.

4.2.2 Induction step: the automatic dag $\mathcal{D}^{i+1}$ Suppose $\mathcal{D}^{i}=(V, E)$ is such that $\mathcal{F}^{i}=$ $\operatorname{unfold}\left(\mathcal{D}^{i}\right)$ is as described in Proposition 16.

We use the notations from Section 4.1.2. We first build another automatic dag $\mathcal{D}^{\prime}$, whose unfolding will comprise (copies of) all the trees $U_{\kappa, x}^{\prime}\left(\kappa \in \mathbb{N}_{+} \cup\{\omega\}, x \in \mathbb{N}_{+}\right)$and $T_{\bar{c} x y}^{\prime}\left(\bar{c} \in \mathbb{N}_{+}^{k}\right.$, $\left.x, y \in \mathbb{N}_{+}\right)$. Recall that the set of roots of $\mathcal{D}^{i}$ is $\otimes_{k+2}\left(a^{+}\right) \cup b^{*} \subseteq V$. The universe of $\mathcal{D}^{\prime}$ consists of the regular language

$$
\left(V \backslash b^{*}\right) \cup\left(\sharp^{+} \otimes b^{*}\right) \cup \sharp_{1}^{+} \sharp_{2}^{*},
$$

where $\sharp_{1} \sharp_{1}$, and $\sharp_{2}$ are new symbols. We have the following edges in $\mathcal{D}^{\prime}$ :

- All edges from $E$ except those with an initial node in $b^{*}$ are present in $\mathcal{D}^{\prime}$.

- $a^{\bar{c} x y} \in V$ is connected to all words of the form $\sharp_{1}^{i} \sharp_{2}^{x-i}$ for $\bar{c} \in \mathbb{N}_{+}^{k}, x, y \in \mathbb{N}_{+}$, and $1 \leq i \leq x$. This ensures that the subtree rooted at $a^{\bar{c} x y}$ gets $x$ new leaves, which are children of the root. Hence $\operatorname{unfold}\left(\mathcal{D}^{\prime}, a^{\bar{c} x y}\right) \cong T_{\bar{c} x y}^{\prime}$.

- $\sharp^{x} \otimes b^{m}$ for $x \in \mathbb{N}_{+}$and $m \in \mathbb{N}$ is connected to (i) all nodes to which $b^{m}$ is connected in $\mathcal{D}^{i}$ and to (ii) all nodes from $\sharp_{1}^{i} \sharp_{2}^{x-i}$ for $1 \leq i \leq x$. This ensures that unfold $\left(\mathcal{D}^{\prime}, \sharp^{x} \otimes b^{m}\right) \cong U_{m, x}^{\prime}$ in case $m \in \mathbb{N}_{+}$and unfold $\left(\mathcal{D}^{\prime}, \sharp^{x} \otimes \varepsilon\right) \cong U_{\omega, x}^{\prime}$.

In summary, $\mathcal{D}^{\prime}$ is a dag, whose unfolding consists of (a copy of) $U_{\omega, x}^{\prime}$ rooted at $\sharp^{x} \otimes \varepsilon, U_{m, x}^{\prime}$ $\left(m \in \mathbb{N}_{+}\right)$rooted at $\sharp^{x} \otimes b^{m}$, and $T_{\bar{c} x y}^{\prime}$ rooted at $a^{\bar{c} x y}$.

From the automatic dag $\mathcal{D}^{\prime}$, we now build in a final step the automatic dag $\mathcal{D}^{i+1}$. This is very similar to the constructions of $\mathcal{D}^{2}$ and $\mathcal{D}^{\prime}$ above. Let $V^{\prime}$ be the universe of $\mathcal{D}^{\prime}$. The universe of $\mathcal{D}^{i+1}$ is the regular language

$$
\otimes_{k}\left(a^{+}\right) \cup b^{*} \cup\left(\$^{*} \otimes V^{\prime}\right)
$$

The edges are as follows:

- For $u, v \in V^{\prime}, \$^{m} \otimes u$ is connected to $\$^{n} \otimes v$ if and only if $m=n$ and $(u, v)$ is an edge of $\mathcal{D}^{\prime}$. This generates $\aleph_{0}$ many copies of $\mathcal{D}^{\prime}$.

$-a^{\bar{c}}$ is connected to every word from $\mathbb{S}^{*} \otimes\left(\left\{a^{\bar{c} x y} \mid x, y \in \mathbb{N}_{+}\right\} \cup\left(\sharp^{+} \otimes b^{+}\right)\right)$. Hence, the tree $\operatorname{unfold}\left(\mathcal{D}^{i+1}, a^{\bar{c}}\right)$ has $\aleph_{0}$ many subtrees isomorphic to $T_{\bar{c} x y}^{\prime}$ for $x, y \in \mathbb{N}_{+}$and $U_{m, x}^{\prime}$ for $x, m \in$ $\mathbb{N}_{+}$. Thus, unfold $\left(\mathcal{D}^{i+1}, a^{\bar{c}}\right) \cong T_{\bar{c}}^{i+1}$. 


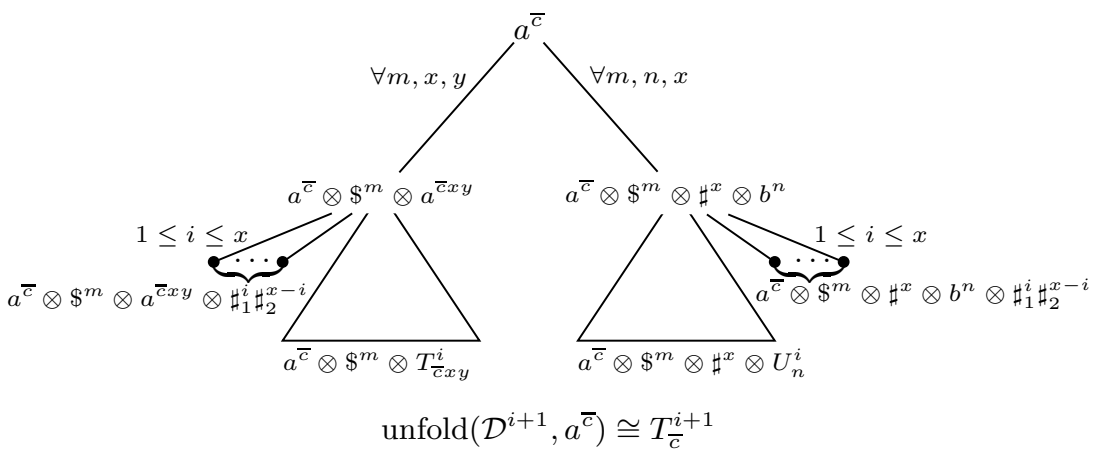

Fig. 4. Automatic presentation of $T_{\bar{c}}^{i+1}$

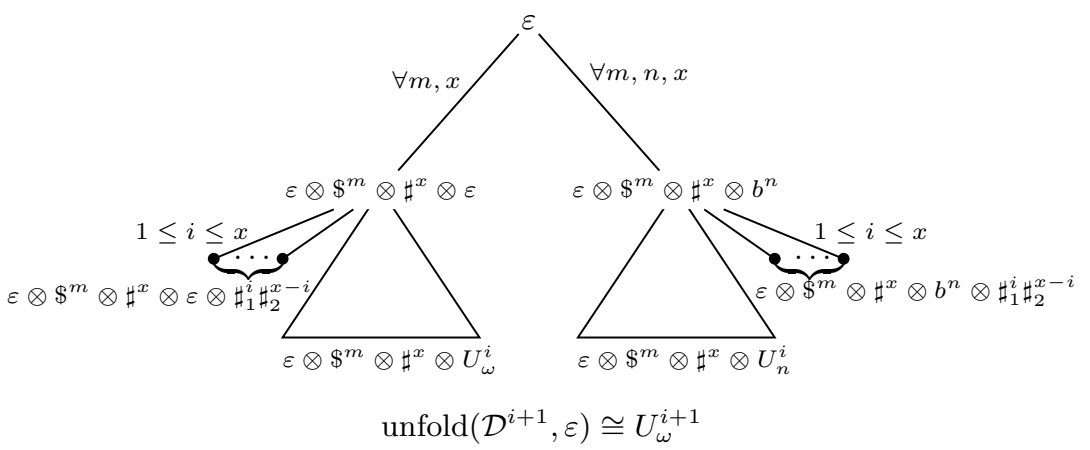

Fig. 5. Automatic presentation of $U_{\omega}^{i+1}$

$-\varepsilon$ is connected to all words from $\$^{*} \otimes\left(\sharp^{+} \otimes b^{*}\right)$. Hence, the tree unfold $\left(\mathcal{D}^{i+1}, \varepsilon\right)$ has $\aleph_{0}$ many subtrees isomorphic to $U_{\kappa, x}^{\prime}$ for all $x \in \mathbb{N}_{+}$and $\kappa \in \mathbb{N}_{+} \cup\{\omega\}$. Thus, unfold $\left(\mathcal{D}^{i+1}, \varepsilon\right) \cong U_{\omega}^{i+1}$.

- $b^{m}\left(m \in \mathbb{N}_{+}\right)$is connected to all words from $\$^{*} \otimes\left(\left(\sharp^{+} \otimes b^{+}\right) \cup\left\{\sharp^{x} \otimes \varepsilon \mid 1 \leq x<m\right\}\right)$. This means that the tree unfold $\left(\mathcal{D}^{i+1}, b^{m}\right)$ has $\aleph_{0}$ many subtrees isomorphic to $U_{m, x}^{\prime}$ for all $m, x \in \mathbb{N}_{+}$and $U_{\omega, x}^{\prime}$ for all $1 \leq x<m$. Hence, $\operatorname{unfold}\left(\mathcal{D}^{i+1}, b^{m}\right) \cong U_{m}^{i+1}$.

See Figure 4, 5, and 6 for the overall construction. This finishes the proof of Proposition 16. Hence we obtain:

Theorem 18. 1. For any $n \geq 2$, the isomorphism problem for automatic trees of height at most $n$ is $\Pi_{2 n-3}^{0}$-complete.

2. The isomorphism problem for the class of automatic trees of finite height is recursively equivalent to $\operatorname{FOTh}(\mathbb{N} ;+, \times)$.

Proof. We first prove the first statement. Containment in $\Pi_{2 n-3}^{0}$ was shown in Proposition 7 . For the hardness, let $P_{n} \subseteq \mathbb{N}_{+}$be any $\Pi_{2 n-3}^{0}$-predicate and let $c \in \mathbb{N}_{+}$. Then, above, we constructed the automatic forest $\mathcal{F}^{n}$ of height $n$. The trees $T_{c}^{n}$ and $U_{\omega}^{n}$ are first-order definable in $\mathcal{F}^{n}$ since they are (isomorphic to) the trees rooted at $a^{c}$ and $\varepsilon$, resp. Hence these two trees are automatic. By Proposition 14, they are isomorphic if and only if $P_{n}(c)$ holds.

We now come to the second statement. Since the proof of Prop. 7 is uniform in the level $n$, we can compute from two automatic trees $T_{1}, T_{2}$ of finite height an arithmetical formula, which is true if and only if $T_{1} \cong T_{2}$. For the other direction, one observes that the height of an automatic tree of finite height can be computed. Then the result follows from the first statement because of the uniformity of its proof.

In fact, we proved a slightly stronger statement: For every $n \geq 2$, there exists a fixed $\Pi_{2 n-3^{-}}^{0}$ complete set $P_{2 n-3} \subseteq \mathbb{N}_{+}$. If we apply our construction, we obtain a fixed automatic forest $\mathcal{F}^{n}$ of height $n$ with the following properties: It is $\Pi_{2 n-3}^{0}$-complete to determine, whether for given $c \in \mathbb{N}_{+}$, the tree rooted at $a^{c}$ in $\mathcal{F}^{n}$ is isomorphic to the tree rooted at $\varepsilon$ in $\mathcal{F}^{n}$. 


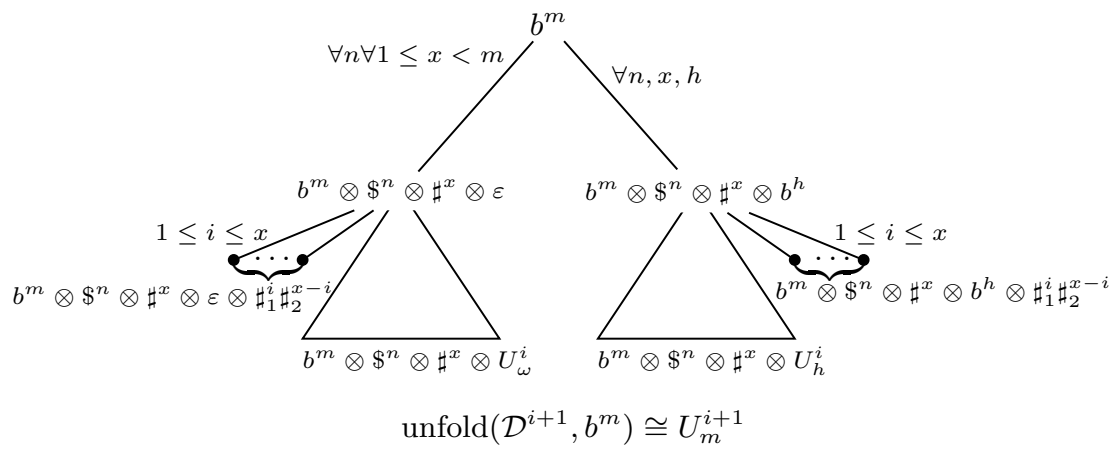

Fig. 6. Automatic presentation of $U_{m}^{i+1}$

\section{$5 \quad$ Recursive trees of finite height}

In this section, we briefly discuss the isomorphism problem for recursive (i.e., computable) trees of finite height.

Theorem 19. For every $n \geq 1$, the isomorphism problem for recursive trees of height at most $n$ is $\Pi_{2 n}^{0}$-complete.

Proof. For the upper bound, let us first assume that $n=1$. Two recursive trees $T_{1}$ and $T_{2}$ of height 1 are isomorphic if and only if: for every $k \geq 0$, there exist at least $k$ nodes in $T_{1}$ if and only if there exist at least $k$ nodes in $T_{2}$. This is a $\Pi_{2}^{0}$-statement. For the inductive step, we can reuse the arguments from the proof of Proposition 17

For the lower bound, we first note that the isomorphism problem for recursive trees of height 1 is $\Pi_{2}^{0}$-complete. It is known that the problem whether a given recursively enumerable set is infinite is $\Pi_{2}^{0}$-complete [19. For a given deterministic Turing-machine $M$, we construct a recursive tree $T(M)$ of height 1 as follows: the set of leaves of $T(M)$ is the set of all accepting computations of $M$. We add a root to the tree and connect the root to all leaves. If $L(M)$ is infinite, then $T(M)$ is isomorphic to the height- 1 tree with infinitely many leaves. If $L(M)$ is finite, then there exists $m \in \mathbb{N}$ such that $T(M)$ is isomorphic to the height-1 tree with $m$ leaves. We can use this construction as the base case for our construction in Section 4.1.2. This yields the lower bound for all $n \geq 1$.

\section{Automatic Linear Orders}

We use $\omega$ to denote the linear order (type of) $(\mathbb{N} ; \leq)$ of the natural numbers and $\mathbf{n}$ to denote the finite linear order (type) of size $n$. Let $I=\left(D_{I} ; \leq_{I}\right)$ be a linear order and let $\mathcal{L}=\left\{L_{i} \mid i \in D_{I}\right\}$ be a class of linear orders, where $L_{i}=\left(D_{i} ; \leq_{i}\right)$ for $i \in D_{I}$. The sum $\sum \mathcal{L}$ is the linear order $\left(\left\{(x, i) \mid i \in D_{I}, x \in D_{i}\right\} ; \leq\right)$ where for all $i, j \in D_{I}, x \in D_{i}$, and $y \in D_{j}$,

$$
(x, i) \leq(y, j) \Longleftrightarrow i<_{I} j \vee\left(i=j \wedge x \leq_{i} y\right) .
$$

We use $L_{1}+L_{2}$ to denote $\sum\left\{L_{i} \mid i \in \mathbf{2}\right\}$. We denote with $L_{1} \cdot L_{2}$ the sum $\sum\left\{L_{1}^{i} \mid i \in L_{2}\right\}$ where $L_{1}^{i} \cong L_{1}$ for every $i \in L_{2}$. An interval of a linear order $L=(D ; \leq)$ is a subset $I \subseteq D$ such that $x, y \in I$ and $x<z<y$ imply $z \in I$.

A well-known example of an automatic linear order is the lexicographic order $\leq_{\operatorname{lex}}$ on a regular language $D$. To define $\leq_{\text {lex }}$, we first need a fixed linear order $<$ on the alphabet $\Sigma$ of $D$. For $w, w^{\prime} \in D$, we say that $w$ is lexicographically less than $w^{\prime}$, denoted by $w<_{\operatorname{lex}} w^{\prime}$, if either $w$ is a proper prefix of $w^{\prime}$ or there exist $x, y, z \in \Sigma^{*}$ and $\sigma, \tau \in \Sigma$ such that $w=x \sigma y, w^{\prime}=x \tau z$ and $\sigma<\tau$. We write $w \leq_{\text {lex }} w^{\prime}$ if either $w=w^{\prime}$ or $w<_{\operatorname{lex}} w^{\prime}$. For convenience, in this paper, we use 
$\leq_{\text {lex }}$ to denote the lexicographic order regardless of the corresponding alphabets and orders on the alphabets. The precise definition of $\leq_{\text {lex }}$ in different occurrences will be clear from the context.

This section is devoted to proving that the isomorphism problem on the class of automatic linear orders is at least as hard as $\operatorname{FOTh}(\mathbb{N} ;+, \times)$. To this end, it suffices to prove (uniformly in n) $\Sigma_{n}^{0}$-hardness for every even $n$. The general plan for this is similar to the proof for trees of finite height: we use Hilbert's $10^{\text {th }}$ problem to handle $\Pi_{1}^{0}$-predicates in several variables and an inductive construction of more complicated linear orders to handle quantifiers, i.e., to proceed from a $\Pi_{2 i-1^{-}}^{0}$ to a $\Sigma_{2 i}^{0}$-predicate (and from a $\Sigma_{2 i}^{0}$ - to a $\Pi_{2 i+1}^{0}$-predicate).

So let $n \geq 1$ be even and let $P_{n}\left(x_{0}\right)$ be a $\Sigma_{n}^{0}$-predicate. For every odd (even) number $1 \leq i<n$, let $P_{i}\left(x_{0}, \ldots, x_{n-i}\right)$ be the $\Pi_{i}^{0}$-predicate $\left(\Sigma_{i}^{0}\right.$-predicate) such that $P_{i+1}\left(x_{0}, \ldots, x_{n-i-1}\right)$ is logically equivalent to $Q x_{n-i}: P_{i}\left(x_{0}, \ldots, x_{n-i}\right)$ where $Q=\exists$ if $i$ is odd and $Q=\forall$ if $i$ is even. We fix these predicates for the rest of Section 6 .

By induction on $1 \leq i \leq n$, we will construct from $\bar{c} \in \mathbb{N}_{+}^{n-i+1}$ the following linear orders:

- a test linear order $L \frac{i}{c}$,

- a linear order $K^{i}$, and

- a set of linear orders $\mathcal{M}^{i}$ such that $\mathcal{M}^{1}=\left\{M_{m}^{1} \mid m \in \mathbb{N}_{+}\right\}$and $\mathcal{M}^{i}$ is the singleton $\left\{M^{i}\right\}$ if $i>1$.

These linear orders will have the following properties:

(P1) $P_{i}(\bar{c})$ holds if and only if $L_{\bar{c}}^{i} \cong K^{i}$.

(P2) $P_{i}(\bar{c})$ does not hold if and only if $L_{\bar{c}}^{i} \cong M$ for some $M \in \mathcal{M}^{i}$.

(P3) The linear order $\omega \cdot \mathbf{i}$ is not isomorphic to any interval of $L_{\bar{c}}, K^{i}, M$ where $M \in \mathcal{M}^{i}$.

In the rest of the section, we will inductively construct $L \frac{i}{c}, K^{i}$, and $\mathcal{M}^{i}$ and prove (P1), (P2), and (P3). The subsequent section is devoted to proving the effective automaticity of these linear orders.

\subsection{Construction of linear orders}

Our construction of linear orders is quite similar to the construction for trees from Section 4.1 . One of the main differences is that in the inductive step for trees, we went from a $\Pi_{i}^{0}$-predicate directly to a $\Pi_{i+2}^{0}$-predicate. Thereby the height of the trees only increased by one. This was crucial in order to get $\Pi_{2 n-3^{-}}^{0}$-completeness for the isomorphism problem for automatic trees of height $n \geq 2$. For automatic linear orders, we split the construction into two inductive steps: in the first step, we go from a $\Pi_{i}^{0}$-predicate $\left(i\right.$ odd) to a $\Sigma_{i+1}^{0}$-predicate, whereas in the second step, we go from a $\Sigma_{i+1}^{0}$-predicate to a $\Pi_{i+2}^{0}$-predicate.

A key technique used in the construction is the shuffle sum of a class of linear orders. Let $I$ be a countable set. A dense $I$-coloring of $\mathbb{Q}$ is a mapping $c: \mathbb{Q} \rightarrow I$ such that for all $x, y \in \mathbb{Q}$ with $x<y$ and all $i \in I$ there exists $x<z<y$ with $c(z)=i$.

Definition 20. Let $\mathcal{L}=\left\{L_{i} \mid i \in I\right\}$ be a set of linear orders with I countable and let $c: \mathbb{Q} \rightarrow I$ be a dense I-coloring of $\mathbb{Q}$. The shuffle sum of $\mathcal{L}$, denoted $\operatorname{Shuf}(\mathcal{L})$, is the linear order $\sum_{x \in \mathbb{Q}} L_{c(x)}$.

In the above definition, the isomorphism type of $\sum_{x \in \mathbb{Q}} L_{c(x)}$ does not depend on the choice of the dense $I$-coloring $c$, see e.g. [20. Hence $\operatorname{Shuf}(\mathcal{L})$ is indeed uniquely defined.

In this section, we will consider classes $\mathcal{L}_{1}$ and $\mathcal{L}_{2}$ of linear orders that we consider as classes of isomorphism types. Therefore, we use the following abbreviations:

- " $L \in \mathcal{L}_{1}$ " denotes that $\mathcal{L}_{1}$ contains a linear order isomorphic to $L$,

- " $\mathcal{L}_{1} \subseteq \mathcal{L}_{2}$ " denotes $\forall L_{1} \in \mathcal{L}_{1} \exists L_{2} \in \mathcal{L}_{2}: L_{1} \cong L_{2}$, and

- " $\mathcal{L}_{1}=\mathcal{L}_{2}$ " abbreviates $\mathcal{L}_{1} \subseteq \mathcal{L}_{2} \subseteq \mathcal{L}_{1}$. 
6.1.1 Induction base: construction of $L \frac{1}{c}, K^{1}$, and $M_{m}^{1}$ Recall from Section 3 that the polynomial function $C(x, y)=(x+y)^{2}+3 x+y$ is injective. For $n_{1}, n_{2} \in \mathbb{N}_{+}$, let $L\left[n_{1}, n_{2}\right]$ be the finite linear order of length $C\left(n_{1}, n_{2}\right)$.

By applying Matiyasevich's theorem, we obtain two polynomials $p_{1}(\bar{x}), p_{2}(\bar{x}) \in \mathbb{N}[\bar{x}]$ in $\ell$ variables, $\ell>n$, such that for all $\bar{c} \in \mathbb{N}_{+}^{n}$, the $\Pi_{1}^{0}$-predicate $P_{1}(\bar{c})$ holds if and only if

$$
\forall \bar{x} \in \mathbb{N}^{\ell-n}: p_{1}(\bar{c}, \bar{x}) \neq p_{2}(\bar{c}, \bar{x}) .
$$

Fix $\bar{c} \in \mathbb{N}_{+}^{n}$ and $m \in \mathbb{N}_{+}$. We define the following four classes of finite linear orders:

$$
\begin{aligned}
\mathcal{L}_{1}^{1}(\bar{c}) & =\left\{L\left[p_{1}(\bar{c}, \bar{x})+x_{\ell+1}, p_{2}(\bar{c}, \bar{x})+x_{\ell+1}\right] \mid \bar{x} \in \mathbb{N}_{+}^{\ell-n}, x_{\ell+1} \in \mathbb{N}_{+}\right\} \\
\mathcal{L}_{2}^{1}(m) & =\left\{L[x+m, x+m] \mid x \in \mathbb{N}_{+}\right\} \\
\mathcal{L}_{3}^{1} & =\left\{L[x+y, x] \mid x, y \in \mathbb{N}_{+}\right\} \\
\mathcal{L}_{4}^{1} & =\left\{L[x, x+y] \mid x, y \in \mathbb{N}_{+}\right\}
\end{aligned}
$$

The linear orders $L \frac{1}{c}, K^{1}$, and $M_{m}^{1}$ are obtained by taking the shuffle sums of unions of the above classes of linear orders:

$$
L_{\bar{c}}^{1}=\operatorname{Shuf}\left(\mathcal{L}_{1}^{1}(\bar{c}) \cup \mathcal{L}_{3}^{1} \cup \mathcal{L}_{4}^{1}\right), \quad K^{1}=\operatorname{Shuf}\left(\mathcal{L}_{3}^{1} \cup \mathcal{L}_{4}^{1}\right), \quad M_{m}^{1}=\operatorname{Shuf}\left(\mathcal{L}_{2}^{1}(m) \cup \mathcal{L}_{3}^{1} \cup \mathcal{L}_{4}^{1}\right) .
$$

The next lemma is needed to prove (P1) and (P2) for the $\Pi_{1}^{0}$-predicate $P_{1}$.

Lemma 21. Suppose $\mathcal{L}_{1}$ and $\mathcal{L}_{2}$ are two countable sets of finite linear orders. Then

$$
\mathcal{L}_{1}=\mathcal{L}_{2} \Longleftrightarrow \operatorname{Shuf}\left(\mathcal{L}_{1}\right) \cong \operatorname{Shuf}\left(\mathcal{L}_{2}\right)
$$

and no interval of $\operatorname{Shuf}\left(\mathcal{L}_{1}\right)$ is isomorphic to $\omega$.

Proof. If $\mathcal{L}_{1}=\mathcal{L}_{2}$, then it is clear that $\operatorname{Shuf}\left(\mathcal{L}_{1}\right) \cong \operatorname{Shuf}\left(\mathcal{L}_{2}\right)$. Conversely, suppose there exists an isomorphism $f$ from $\operatorname{Shuf}\left(\mathcal{L}_{1}\right)$ to $\operatorname{Shuf}\left(\mathcal{L}_{2}\right)$. We prove below that $\mathcal{L}_{1}=\mathcal{L}_{2}$. By symmetry we only need to prove $\mathcal{L}_{1} \subseteq \mathcal{L}_{2}$.

Note that for $i \in\{1,2\}$, $\operatorname{Shuf}\left(\mathcal{L}_{i}\right)$ is obtained by replacing each $q \in \mathbb{Q}$ with some linear order $L_{q}^{i}$ (whose type is) contained in $\mathcal{L}_{i}$. For every $q \in \mathbb{Q}$, if $f\left(L_{q}^{1}\right)$ contains elements from $L_{p}^{2}$ and $L_{p^{\prime}}^{2}$ for some $p<p^{\prime}$, then $f\left(L_{q}^{1}\right)$ is infinite which is impossible. Therefore $f$ maps $L_{q}^{1}$ into $L_{p}^{2}$ for some $p \in \mathbb{Q}$. Using the same argument with $f$ replaced by $f^{-1}$, we can also prove that $f^{-1}$ maps $L_{p}^{2}$ into $L_{q}^{1}$. Hence $L_{q}^{1} \cong L_{p}^{2}$. This means that for all $L \in \mathcal{L}_{1}$, there is $L^{\prime} \in \mathcal{L}_{2}$ such that $L \cong L^{\prime}$. Therefore $\mathcal{L}_{1} \subseteq \mathcal{L}_{2}$.

If $x_{1}<x_{2}<\cdots$ in $\operatorname{Shuf}\left(\mathcal{L}_{1}\right)$, then there are $p<p^{\prime}$ in $\mathbb{Q}$ and $k<\ell$ in $\mathbb{N}_{+}$such that $x_{k} \in L_{p}^{1}$ and $x_{\ell} \in L_{p^{\prime}}^{1}$. But then the interval $\left[x_{k}, x_{\ell}\right]$ is infinite. Hence no interval in $\operatorname{Shuf}\left(\mathcal{L}_{1}\right)$ is isomorphic to $\omega$.

The next lemma states (P1) and (P2) for $i=1$ :

Lemma 22. For any $\bar{c} \in \mathbb{N}_{+}^{n}$, we have:

(1) $P_{1}(\bar{c})$ holds $\Longleftrightarrow L \frac{1}{c} \cong K^{1}$.

(2) $P_{1}(\bar{c})$ does not hold $\Longleftrightarrow \exists m \in \mathbb{N}_{+}: L_{\bar{c}}^{1} \cong M_{m}^{1}$.

Proof. For (1), we have

$$
\begin{aligned}
P_{1}(\bar{c}) & \Longleftrightarrow \quad \forall \bar{x} \in \mathbb{N}_{+}^{\ell-n}: p_{1}(\bar{c}, \bar{x}) \neq p_{2}(\bar{c}, \bar{x}) \\
& \Longleftrightarrow \quad \forall \bar{x} \in \mathbb{N}_{+}^{\ell-n}, x_{\ell+1} \in \mathbb{N}_{+}: p_{1}(\bar{c}, \bar{x})+x_{\ell+1} \neq p_{2}(\bar{c}, \bar{x})+x_{\ell+1} \\
& \Longleftrightarrow \quad \forall \bar{x} \in \mathbb{N}_{+}^{\ell-n}, x_{\ell+1} \in \mathbb{N}_{+}: L\left[p_{1}(\bar{c}, \bar{x})+x_{\ell+1}, p_{2}(\bar{c}, \bar{x})+x_{\ell+1}\right] \in \mathcal{L}_{3}^{1} \cup \mathcal{L}_{4}^{1} \\
& \Longleftrightarrow \quad \mathcal{L}_{1}^{1}(\bar{c}) \cup \mathcal{L}_{3}^{1} \cup \mathcal{L}_{4}^{1}=\mathcal{L}_{3}^{1} \cup \mathcal{L}_{4}^{1} \\
& \Longleftrightarrow L_{\bar{c}}^{\text {Lemma } 21} \cong K^{1} .
\end{aligned}
$$


For (2), we get

$$
\begin{aligned}
& \neg P_{1}(\bar{c}) \quad \Longleftrightarrow \quad \exists \bar{x} \in \mathbb{N}_{+}^{\ell-n}: p_{1}(\bar{c}, \bar{x})=p_{2}(\bar{c}, \bar{x}) \\
& \Longleftrightarrow \quad \exists m \in \mathbb{N}_{+}: L[m+1, m+1] \in \mathcal{L}_{1}^{1}(\bar{c}) \\
& \Longleftrightarrow \quad \exists m \in \mathbb{N}_{+}:\left(\forall k>m: L[k, k] \in \mathcal{L}_{1}^{1}(\bar{c}) \wedge \forall 1 \leq k \leq m: L[k, k] \notin \mathcal{L}_{1}^{1}(\bar{c})\right) \\
& \Longleftrightarrow \quad \exists m \in \mathbb{N}_{+}: \mathcal{L}_{1}^{1}(\bar{c}) \cup \mathcal{L}_{3}^{1} \cup \mathcal{L}_{4}^{1}=\mathcal{L}_{2}^{1}(m) \cup \mathcal{L}_{3}^{1} \cup \mathcal{L}_{4}^{1} \\
& \stackrel{\text { Lemma 21] }}{\Longleftrightarrow} \exists m \in \mathbb{N}_{+}: L \frac{1}{c} \cong M_{m}^{1} \text {. }
\end{aligned}
$$

Since $L \frac{1}{c}, K^{1}$, and $M_{m}^{1}$ are shuffle sums, they satisfy (P3) by Lemma 21, This finishes the construction for the base case.

6.1.2 First induction step: from $\boldsymbol{P}_{\boldsymbol{i}}$ to $\boldsymbol{P}_{\boldsymbol{i}+\mathbf{1}}$ for $\boldsymbol{i}$ odd Suppose $i \geq 1$ is an odd number. For notational simplicity, we write $k$ for $n-i$. Thus, $P_{i+1}$ is a $k$-ary predicate and $P_{i}$ is a $(k+1)$ ary one. For all $\bar{c} \in \mathbb{N}_{+}^{k}, P_{i+1}(\bar{c})$ is logically equivalent to $\exists x: P_{i}(\bar{c}, x)$. Applying the inductive hypothesis, for any $\bar{c} \in \mathbb{N}_{+}^{k}$ and $x \in \mathbb{N}_{+}$, we obtain linear orders $L_{\bar{c} x}^{i}, K^{i}$, and the set $\mathcal{M}^{i}$ such that

$-P_{i}(\bar{c}, x)$ holds if and only if $L_{\bar{c} x}^{i} \cong K^{i}$,

- $P_{i}(\bar{c}, x)$ does not hold if and only if $L_{\bar{c} x}^{i} \cong M$ for some $M \in \mathcal{M}^{i}$, and

- $\omega \cdot \mathbf{i}$ is not isomorphic to any interval of $L_{\bar{c} x}^{i}, K^{i}$, or $M$ where $M \in \mathcal{M}^{i}$.

Fix $\bar{c} \in \mathbb{N}_{+}^{k}$. We define the following classes of linear orders:

$$
\mathcal{L}_{1}^{i+1}(\bar{c})=\left\{\omega \cdot \mathbf{i}+L_{\bar{c} x}^{i} \mid x \in \mathbb{N}_{+}\right\}, \quad \mathcal{L}_{2}^{i+1}=\left\{\omega \cdot \mathbf{i}+M \mid M \in \mathcal{M}^{i}\right\}, \quad \mathcal{L}_{3}^{i+1}=\left\{\omega \cdot \mathbf{i}+K^{i}\right\} .
$$

The linear orders $L_{\bar{c}}^{i+1}, K^{i+1}$, and $M^{i+1}$ are defined as shuffle sums of unions of the above classes of linear orders:

$$
L_{\bar{c}}^{i+1}=\operatorname{Shuf}\left(\mathcal{L}_{1}^{i+1}(\bar{c}) \cup \mathcal{L}_{2}^{i+1}\right), \quad K^{i+1}=\operatorname{Shuf}\left(\mathcal{L}_{2}^{i+1} \cup \mathcal{L}_{3}^{i+1}\right), \quad M^{i+1}=\operatorname{Shuf}\left(\mathcal{L}_{2}^{i+1}\right) .
$$

Recall that the set $\mathcal{M}^{i}$ is a singleton for $i>1$, consisting of $M^{i}$. The next lemma can be proved similarly as Lemma 21

Lemma 23. Suppose $\mathcal{L}_{1}$ and $\mathcal{L}_{2}$ are two countable classes of linear orders such that each $L \in$ $\mathcal{L}_{1} \cup \mathcal{L}_{2}$ is isomorphic to a linear order of the form $\omega \cdot \mathbf{i}+K$, where $\omega \cdot \mathbf{i}$ is not isomorphic to any interval of $K$. Then

$$
\mathcal{L}_{1}=\mathcal{L}_{2} \Longleftrightarrow \operatorname{Shuf}\left(\mathcal{L}_{1}\right) \cong \operatorname{Shuf}\left(\mathcal{L}_{2}\right) .
$$

If $\operatorname{Shuf}\left(\mathcal{L}_{1}\right)$ contains an interval isomorphic to $\omega \cdot(\mathbf{i}+\mathbf{1})$, then there is a linear order $K$ with $\omega \cdot(\mathbf{i}+\mathbf{1})+K \in \mathcal{L}_{1}$.

Proof. If $\mathcal{L}_{1}=\mathcal{L}_{2}$, then it is clear that $\operatorname{Shuf}\left(\mathcal{L}_{1}\right) \cong \operatorname{Shuf}\left(\mathcal{L}_{2}\right)$. Conversely, suppose $f$ is an isomorphism from $\operatorname{Shuf}\left(\mathcal{L}_{1}\right)$ to $\operatorname{Shuf}\left(\mathcal{L}_{2}\right)$. We prove that $\mathcal{L}_{1}=\mathcal{L}_{2}$. By symmetry we only need to prove that $\mathcal{L}_{1} \subseteq \mathcal{L}_{2}$.

Say $\mathcal{L}_{j}=\left\{L_{j, s} \mid s \in \mathbb{N}\right\}$ for $j \in\{1,2\}$. Intuitively, for $j \in\{1,2\}$, $\operatorname{Shuf}\left(\mathcal{L}_{j}\right)$ can be viewed as obtained by replacing each $q \in \mathbb{Q}$ with a linear order $L(j, q) \cong L_{j, c(q)}$, where $c$ is a dense $\mathbb{N}$-coloring. Fix $q \in \mathbb{Q}$. Suppose $f(L(1, q))$ contains elements in $L(2, p)$ and $L\left(2, p^{\prime}\right)$ for $p, p^{\prime} \in \mathbb{Q}$ with $p<p^{\prime}$. Then in $f(L(1, q))$ there are infinitely many disjoint intervals that are isomorphic to $\omega \cdot \mathbf{i}$, while in $L(1, q)$ there is exactly one such interval, a contradiction. Therefore $f$ maps $L(1, q)$ into $L(2, p)$ for some $p \in \mathbb{Q}$.

If $f(L(1, q)) \varsubsetneqq L(2, p)$, then $f^{-1}(L(2, p))$ contains an element $x \notin L(1, q)$. The argument from the previous paragraph with $f$ replaced by $f^{-1}$ again leads to a contradiction. Therefore $f(L(1, q))=L(2, p)$. This means that for all $L \in \mathcal{L}_{1}$, there is $L^{\prime} \in \mathcal{L}_{2}$ such that $L \cong L^{\prime}$ and the lemma is proved. 
Let $I \cong \omega \cdot(\mathbf{i}+\mathbf{1})$ be some interval in $\operatorname{Shuf}\left(\mathcal{L}_{1}\right)$. First suppose there are $p<r$ in $\mathbb{Q}$ such that $I$ intersects $L(1, p)$ and $L(1, r)$. But then $L(1, q) \subseteq I$ for all $p<q<r$, implying that $(\mathbb{Q}, \leq)$ embeds into $I \cong \omega \cdot(\mathbf{i}+\mathbf{1})$ which is impossible. Hence there is some $q \in \mathbb{Q}$ with $I \subseteq L(1, q) \in \mathcal{L}_{1}$. Then there is a linear order $K$ such that $L(1, q)=\omega \cdot \mathbf{i}+K$. Since $\omega \cdot \mathbf{i}$ (let alone $\omega \cdot(\mathbf{i}+\mathbf{1})$ ) is no interval in $K$, the interval $I$ has to intersect the initial segment $\omega \cdot \mathbf{i}$ of $L(1, q)$. But then $\omega$ has to be an initial segment of $K$, i.e., $L(1, q)=\omega \cdot(\mathbf{i}+\mathbf{1})+K^{\prime}$ for some linear order $K^{\prime}$.

Now notice that $\omega \cdot(\mathbf{i}+\mathbf{1})$ is not isomorphic to any interval of $L_{\bar{c}}^{i+1}, K^{i+1}$, or $M^{i+1}$ (each of the orders $L_{\bar{c} x}^{i}, K^{i}$, and $M \in \mathcal{M}^{i}$ is a shuffle sum and therefore does not start with $\omega$ ). Hence (P3) holds for $i+1$. Furthermore, the following holds:

$$
\begin{aligned}
& P_{i+1}(\bar{c}) \quad \Longleftrightarrow \quad \exists x \in \mathbb{N}_{+}: P_{i+1}(\bar{c}, x) \\
& \Longleftrightarrow \quad \exists x \in \mathbb{N}_{+}: L_{\bar{c} x}^{i} \cong K^{i} \\
& \Longleftrightarrow \quad \mathcal{L}_{3}^{i+1} \subseteq \mathcal{L}_{1}^{i+1}(\bar{c}) \\
& \Longleftrightarrow \quad \mathcal{L}_{1}^{i+1}(\bar{c}) \cup \mathcal{L}_{2}^{i+1}=\mathcal{L}_{2}^{i+1} \cup \mathcal{L}_{3}^{i+1} \\
& \stackrel{\text { Lemma } 23}{\Longleftrightarrow} L_{\bar{c}}^{i+1} \cong K^{i+1} \\
& \neg P_{i+1}(\bar{c}) \quad \Longleftrightarrow \quad \forall x \in \mathbb{N}_{+}: \neg P_{i+1}(\bar{c}, x) \\
& \Longleftrightarrow \quad \forall x \in \mathbb{N}_{+} \exists M \in \mathcal{M}^{i}: L_{\bar{c} x}^{i} \cong M \\
& \Longleftrightarrow \quad \mathcal{L}_{1}^{i+1}(\bar{c}) \cup \mathcal{L}_{2}^{i+1}=\mathcal{L}_{2}^{i+1} \\
& \stackrel{\text { Lemma } 23}{\Longleftrightarrow} L_{\bar{c}}^{i+1} \cong M^{i+1}
\end{aligned}
$$

We have shown (P1) and (P2) for $i+1$ in case $i$ is odd.

6.1.3 Second induction step: from $\boldsymbol{P}_{\boldsymbol{i}}$ to $\boldsymbol{P}_{\boldsymbol{i}+\boldsymbol{1}}$ for $\boldsymbol{i}$ even Let $i \geq 1$ be even and consider the $\Pi_{i+1}^{0}$-predicate $P_{i+1}$. Again, we write $k$ for $n-i$. For all $\bar{c} \in \mathbb{N}_{+}^{k}, P_{i+1}(\bar{c})$ is logically equivalent to $\forall x: P_{i}(\bar{c}, x)$. Since $i$ is even, we must have $i \geq 2$. Therefore the set $\mathcal{M}^{i}$ is a singleton, consisting of the linear order $M^{i}$.

Fix $\bar{c} \in \mathbb{N}_{+}^{k}$. Define the classes of linear orders $\mathcal{L}_{1}^{i+1}(\bar{c}), \mathcal{L}_{2}^{i+1}$, and $\mathcal{L}_{3}^{i+1}$ using the same definition as in (13). The linear orders $L_{\bar{c}}^{i+1}, K^{i+1}$, and $M^{i+1}$ are defined as follows:

$$
L_{\bar{c}}^{i+1}=\operatorname{Shuf}\left(\mathcal{L}_{1}^{i+1}(\bar{c}) \cup \mathcal{L}_{3}^{i+1}\right), \quad K^{i+1}=\operatorname{Shuf}\left(\mathcal{L}_{3}^{i+1}\right), \quad M^{i+1}=\operatorname{Shuf}\left(\mathcal{L}_{2}^{i+1} \cup \mathcal{L}_{3}^{i+1}\right) .
$$

Again, $\omega \cdot(\mathbf{i}+\mathbf{1})$ is not isomorphic to any interval of $L_{\bar{c}}^{i+1}, K^{i+1}$, or $M^{i+1}$. Hence (P3) holds for $i+1$. Furthermore, the following holds:

$$
\begin{aligned}
& P_{i+1}(\bar{c}) \quad \Longleftrightarrow \quad \forall x \in \mathbb{N}_{+}: P_{i}(\bar{c}, x) \\
& \Longleftrightarrow \quad \forall x \in \mathbb{N}_{+}: L_{\bar{c} x}^{i} \cong K^{i} \\
& \Longleftrightarrow \quad \mathcal{L}_{1}^{i+1}(\bar{c}) \cup \mathcal{L}_{3}^{i+1}=\mathcal{L}_{3}^{i+1} \\
& \stackrel{\text { Lemma } 23}{\Longleftrightarrow} L_{\bar{c}}^{i+1} \cong K^{i+1} \\
& \neg P_{i+1}(\bar{c}) \quad \Longleftrightarrow \quad \exists x \in \mathbb{N}_{+}: \neg P_{i}(\bar{c}, x) \\
& \Longleftrightarrow \quad \exists x \in \mathbb{N}_{+}: L_{\bar{c} x}^{i} \cong M^{i} \\
& \Longleftrightarrow \quad \mathcal{L}_{1}^{i+1}(\bar{c}) \cup \mathcal{L}_{3}^{i+1}=\mathcal{L}_{2}^{i+1} \cup \mathcal{L}_{3}^{i+1} \\
& \stackrel{\text { Lemma }}{\Longleftrightarrow} L_{\bar{c}}^{i+1} \cong M^{i+1}
\end{aligned}
$$

We have shown (P1) and (P2) for $i+1$ in case $i$ is even. This finishes the construction and proof for $(\mathrm{P} 1),(\mathrm{P} 2)$, and $(\mathrm{P} 3)$ in the inductive step.

\subsection{Automaticity}

To construct automatic presentations of the linear orders from the previous section, we first fix some notations. For $\bar{c}=\left(c_{1}, \ldots, c_{k}\right) \in \mathbb{N}_{+}^{k}$ and a symbol $a$, we re-define $a^{\bar{c}}$ as the word

$$
a^{c_{1}} \sharp \cdots a^{c_{k}} \sharp \in\{a, \sharp\}^{*} \text {. }
$$


Recall that Lemma 2 described a way to represent a polynomial $p(\bar{x}) \in \mathbb{N}[\bar{x}]$ in $k$ variables using the number of accepting runs of an automaton $\mathcal{A}[p(\bar{x})]$. The next lemma re-states Lemma 2 with respect to the new definition of $a^{\bar{c}}$.

Lemma 24. From a polynomial $p(\bar{x}) \in \mathbb{N}[\bar{x}]$ in $k$ variables, one can effectively construct a nondeterministic automaton $\mathcal{A}[p(\bar{x})]$ on alphabet $\{a, \sharp\}$ such that $L(\mathcal{A}[p(\bar{x})])=\left(a^{+} \sharp\right)^{k}$ and for all $\bar{c} \in \mathbb{N}_{+}^{k}: \mathcal{A}[p(\bar{x})]$ has exactly $p(\bar{c})$ accepting runs on input $a^{\bar{c}}$.

Proof. We use the same proof as for Lemma 2. The only difference is when the polynomial $p\left(x_{1}, \ldots, x_{k}\right)$ is of the form $x_{i}$ for some $i \in\{1, \ldots, k\}$. In this case, the automaton $\mathcal{A}\left[x_{i}\right]$ is $(S, I, \Delta, F)$ where $S=\left\{q_{0}, q_{1}, \ldots q_{k}, q_{i}^{\prime}\right\}, I=\left\{q_{0}\right\}, F=\left\{q_{k}\right\}$ and the transition relation $\Delta$ is

$$
\Delta=\left\{\left(q_{j-1}, \sharp, q_{j}\right) \mid 1 \leq j \leq k, j \neq i\right\} \cup\{(q, a, q) \mid q \in S\} \cup\left\{\left(q_{i-1}, a, q_{i}^{\prime}\right),\left(q_{i}^{\prime}, \sharp, q_{i}\right)\right\} .
$$

It is easy to see that $L\left(\mathcal{A}\left[x_{i}\right]\right)=\left(a^{+} \sharp\right)^{k}$ and $\mathcal{A}\left[x_{i}\right]$ has exactly $c_{i}$ accepting runs on input $a^{\bar{c}}$ where $\bar{c} \in \mathbb{N}_{+}^{k}$.

From now on, when referring to $\mathcal{A}[p(\bar{x})]$, we always assume it is defined in the sense of Lemma 24 (as opposed to Lemma 2). Let $\mathcal{A}$ be a non-deterministic finite automaton over the alphabet $\Sigma$ and let $\Delta$ be the transition relation of $\mathcal{A}$. Recall the definition of the automaton $\operatorname{Run}_{\mathcal{A}}$ and the projection morphism $\pi: \Delta^{*} \rightarrow \Sigma^{*}$ from Section 3 . Then, $\operatorname{Run}_{\mathcal{A}}$ is an automaton over the alphabet $\Delta$. Assume that a lexicographic order $\leq_{\operatorname{lex}}$ has been defined on each of $\Sigma^{*}$ and $\Delta^{*}$. Define the automatic linear order $\sqsubseteq$ on $L\left(\operatorname{Run}_{\mathcal{A}}\right)$ such that for all $w, w^{\prime} \in L\left(\operatorname{Run}_{\mathcal{A}}\right)$ :

$$
w \sqsubseteq w^{\prime} \Longleftrightarrow \pi(w)<_{\operatorname{lex}} \pi\left(w^{\prime}\right) \vee\left(\pi(w)=\pi\left(w^{\prime}\right) \wedge w \leq_{\operatorname{lex}} w^{\prime}\right) .
$$

Let $\Sigma_{i}$ be the alphabet $\left\{\sharp, \$_{1}, \ldots, \$_{i-1}, \$, 0,1, a, b_{1}, b_{2}, b_{3}\right\}$. Fix the order $<$ on $\Sigma_{i}$ such that

$$
\$<\$_{1}<\cdots<\$_{i-1}<0<\sharp<a<b_{1}<b_{2}<b_{3}<1 .
$$

For any automaton $\mathcal{A}$ over $\Sigma_{i}$, fix an arbitrary order on the transition relation $\Delta$ of $\mathcal{A}$. Let $\leq_{\text {lex }}$ be the lexicographic orders on $\Sigma_{i}^{*}$ and $\Delta^{*}$ defined with respect to these orders, respectively. From now on, we will always let $\sqsubseteq$ be the linear order as defined in (15) with respect to $\leq_{\text {lex }}$. For a regular language $L \subseteq \Sigma^{*}$ let first $(L)=\left\{a \in \Sigma \mid \exists w \in \Sigma^{*}: a w \in L\right\}$. For $u \in \Sigma^{*}$, we use $L[u]$ to denote the language $u \Sigma^{*} \cap L$. Technically, in this section we prove by induction on $i$ the following statement:

Proposition 25. We can compute automata $\mathcal{A}^{i}$ over $\Sigma_{i}$ such that:

(1) $L\left(\mathcal{A}^{1}\right)=\left(\left(a^{+} \sharp\right)^{n} \cup b_{1}^{+} \sharp \cup b_{2} \sharp\right) \$ R$ for some regular language $R \subseteq \Sigma_{1}^{+}$

(2) If $i>1$, then $L\left(\mathcal{A}^{i}\right)=\left(\left(a^{+} \sharp\right)^{n-i+1} \cup b_{1} \sharp \cup b_{2} \sharp\right) \$ R$ for some regular language $R \subseteq \Sigma_{i}^{+}$

(3) $L \frac{i}{\bar{c}} \cong\left(\pi^{-1}\left(L\left(\mathcal{A}^{i}\right)\left[\bar{a}^{\bar{c}}\right]\right) \cap L\left(\operatorname{Run}_{\mathcal{A}^{i}}\right) ; \sqsubseteq\right)$ for $\bar{c} \in \mathbb{N}_{+}^{n-i+1}$

(4) $M_{m}^{1} \cong\left(\pi^{-1}\left(L\left(\mathcal{A}^{1}\right)\left[b_{1}^{m} \sharp\right]\right) \cap L\left(\operatorname{Run}_{\mathcal{A}^{1}}\right)\right.$; $\left.\sqsubseteq\right)$ for $m \in \mathbb{N}_{+}$

(5) $M^{i} \cong\left(\pi^{-1}\left(L\left(\mathcal{A}^{i}\right)\left[b_{1} \sharp\right]\right) \cap L\left(\operatorname{Run}_{\mathcal{A}^{i}}\right)\right.$; ) for $i>1$

(6) $K^{i} \cong\left(\pi^{-1}\left(L\left(\mathcal{A}^{i}\right)\left[b_{2} \sharp\right]\right) \cap L\left(\operatorname{Run}_{\mathcal{A}^{i}}\right)\right.$;

Moreover, in (1) and (2) we have first $(R) \subseteq\{0,1\}$.

6.2.1 Effective automaticity of shuffle sums This section shows that we can construct an automatic presentation of the shuffle sum of a class of automatic linear orders that are presented in some specific way. For a regular language $D$ over an alphabet, which does neither contain 0 nor 1 , let $\sigma(D)=\left(\{0,1\}^{*} 1 D\right)^{+}$.

Lemma 26. Let $\mathcal{A}$ be an automaton such that $L(\mathcal{A})=E D \$ F$ for regular languages $E, D \subseteq$ $\left\{a, b_{1}, b_{2}, b_{3}, \sharp\right\}^{*}$ and $F \subseteq \Sigma_{i}^{*}$ (for some $1 \leq i \leq n$ ). We can effectively compute an automaton $\sigma(\mathcal{A}, E)$ such that $L(\sigma(\mathcal{A}, E))=E \$ \sigma(D) \$ F$ and for all $u \in E$ :

$$
\left(\pi^{-1}(u \$ \sigma(D) \$ F) \cap L\left(\operatorname{Run}_{\sigma(\mathcal{A}, E)}\right) ; \sqsubseteq\right) \cong \operatorname{Shuf}\left(\left\{\left(\pi^{-1}(u v \$ F) \cap L\left(\operatorname{Run}_{\mathcal{A}}\right) ; \sqsubseteq\right) \mid v \in D\right\}\right) .
$$


Proof. Suppose $\mathcal{A}=\left(S, I, \Delta, S_{f}\right)$. Let $\Gamma=\left\{a, b_{1}, b_{2}, b_{3}, \sharp\right\}$. We first define the automaton

$$
\mathcal{A}^{\prime}=\left(S \times\{1,2, \text { loop }\}, I \times\{1\}, \Delta^{\prime}, S_{f} \times\{2\}\right) .
$$

The transition function $\Delta^{\prime}$ of $\mathcal{A}^{\prime}$ is defined as follows:

$$
\begin{aligned}
\Delta^{\prime}= & \{((q, 1), \alpha,(p, 1)) \mid(q, \alpha, p) \in \Delta, \alpha \in \Gamma\} \cup \\
& \{((q, 1), \$,(q, \text { loop })) \mid q \in S\} \cup \\
& \{((q, \text { loop }), \alpha,(q, \text { loop })) \mid \alpha \in \Gamma \cup\{0,1\}\} \cup \\
& \{((q, \text { loop }), 1,(q, 2)) \mid q \in S\} \cup \\
& \{((q, 2), \alpha,(p, 2)) \mid(q, \alpha, p) \in \Delta\}
\end{aligned}
$$

Intuitively, $\mathcal{A}^{\prime}$ consists of two copies of $\mathcal{A}$ whose state spaces are $S \times\{1\}$ and $S \times\{2\}$. The automaton $\mathcal{A}^{\prime}$ runs by starting simulating $\mathcal{A}$ on the first copy. When the first $\$$ is read, it stops the simulation. For this, the automaton stores the state $q$ by moving to the "looping state" ( $q$, loop). The automaton will stay in ( $q$, loop) unless 1 is read, in which case, it may "guess" that it reads the last 1 before the second $\$$ in the input. If so, it goes out of ( $q$, loop) and continues the simulation in the second copy of $\mathcal{A}$ and accepts the input word if the run stops at a final state. If the guess was not correct and there is another 1 before the second $\$$ in the input, then the run will necessarily reject.

It is easy to see that for all $u_{1}, u_{2} \in \Gamma^{*}, v \in(\Gamma \cup\{0,1\})^{*} 1$ and $u_{3} \in F$, the number of accepting runs of $\mathcal{A}^{\prime}$ on $u_{1} \$ v u_{2} \$ u_{3}$ is the same as the number of accepting runs of $\mathcal{A}$ on $u_{1} u_{2} \$ u_{3}$, i.e.,

$$
\left|L\left(\operatorname{Run}_{\mathcal{A}^{\prime}}\right) \cap \pi^{-1}\left(u_{1} \$ v u_{2} \$ u_{3}\right)\right|=\left|L\left(\operatorname{Run}_{\mathcal{A}}\right) \cap \pi^{-1}\left(u_{1} u_{2} \$ u_{3}\right)\right| .
$$

Let

$$
\sigma(\mathcal{A}, E)=E \$ \sigma(D) \$ F \cap \mathcal{A}^{\prime} .
$$

Note that $L(\sigma(\mathcal{A}, E))=E \$ \sigma(D) \$ F$. Also, for any $u_{1} \in E, v \in\left(\{0,1\}^{*} 1 D\right)^{*}\{0,1\}^{*} 1, u_{2} \in D$, and $u_{3} \in F$, the number of accepting runs of $\sigma(\mathcal{A}, E)$ on $u_{1} \$ v u_{2} \$ u_{3}$ equals the number of accepting runs of $\mathcal{A}^{\prime}$ on $u_{1} \$ v u_{2} \$ u_{3}$, which is, by (17), equal to the number of accepting runs of $\mathcal{A}$ on $u_{1} u_{2} \$ u_{3}$. Hence, we have

$$
\left|L\left(\operatorname{Run}_{\sigma(\mathcal{A}, E)}\right) \cap \pi^{-1}\left(u_{1} \$ v u_{2} \$ u_{3}\right)\right|=\left|L\left(\operatorname{Run}_{\mathcal{A}}\right) \cap \pi^{-1}\left(u_{1} u_{2} \$ u_{3}\right)\right| .
$$

We prove the following claim.

Claim 1. For all $u_{1} \in E, v \in\left(\{0,1\}^{*} 1 D\right)^{*}\{0,1\}^{*} 1$ and $u_{2} \in D$,

$$
\left(\pi^{-1}\left(u_{1} \$ v u_{2} \$ F\right) \cap L\left(\operatorname{Run}_{\sigma(\mathcal{A}, E)}\right) ; \sqsubseteq\right) \cong\left(\pi^{-1}\left(u_{1} u_{2} \$ F\right) \cap L\left(\operatorname{Run}_{\mathcal{A}}\right) ; \sqsubseteq\right) .
$$

For $u \in F$, let $L(u)=\left(\pi^{-1}\left(u_{1} u_{2} \$ u\right) \cap L\left(\operatorname{Run}_{\mathcal{A}}\right) ; \sqsubseteq\right)$. Note that this is a finite linear order. Consider the linear order $\left(F ; \leq_{\text {lex }}\right)$. By definition of $\sqsubseteq$,

$$
\left(\pi^{-1}\left(u_{1} u_{2} \$ F\right) \cap L\left(\operatorname{Run}_{\mathcal{A}}\right) ; \sqsubseteq\right) \cong \sum_{u \in F} L(u) .
$$

By (18), $L(u) \cong\left(\pi^{-1}\left(u_{1} \$ v u_{2} \$ u\right) \cap L\left(\operatorname{Run}_{\sigma(\mathcal{A}, E)}\right) ; \sqsubseteq\right)$. By definition of $\sqsubseteq$ again,

$$
\begin{aligned}
\left(\pi^{-1}\left(u_{1} \$ v u_{2} \$ F\right) \cap L\left(\operatorname{Run}_{\sigma(\mathcal{A}, E)}\right) ; \sqsubseteq\right) & \cong \sum_{u \in F}\left(\pi^{-1}\left(u_{1} \$ v u_{2} \$ u\right) \cap L\left(\operatorname{Run}_{\sigma(\mathcal{A}, E)}\right) ; \sqsubseteq\right) \\
& \cong \sum_{u \in F} L(u) \\
& \cong\left(\pi^{-1}\left(u_{1} u_{2} \$ F\right) \cap L\left(\operatorname{Run}_{\mathcal{A}}\right) ; \sqsubseteq\right) .
\end{aligned}
$$

This proves Claim 1. 
Let $c: \sigma(D) \rightarrow D$ be the function such that

$$
\forall x \in\left(\{0,1\}^{*} 1 D\right)^{*}\{0,1\}^{*} 1 \forall u \in D: c(x u)=u .
$$

Claim 2. $\left(\sigma(D) ; \leq_{\text {lex }}\right) \cong(\mathbb{Q} ; \leq)$ and the function $c$ is a dense $D$-coloring of $\left(\sigma(D) ; \leq_{\text {lex }}\right)$.

First, for every $w=x 1 u \in \sigma(D)$ with $x \in\left(\{0,1\}^{*} 1 D\right)\{0,1\}^{*}$ and $u \in D$, we have

$$
x 01 u<_{\operatorname{lex}} w<_{\operatorname{lex}} x 11 u .
$$

Hence, $\left(\sigma(D) ; \leq_{\text {lex }}\right)$ does not have a smallest or largest element. It remains to show that the linear order $\left(\sigma(D) ; \leq_{\text {lex }}\right)$ is densely $D$-colored by $c$ (this implies that $\left(\sigma(D) ; \leq_{\text {lex }}\right)$ is dense and hence, by Cantor's theorem, isomorphic to $(\mathbb{Q} ; \leq))$. Consider two words $w_{1}, w_{2} \in \sigma(D)$ such that $w_{1}<_{\operatorname{lex}} w_{2}$. There are two cases.

Case 1. $w_{1}=x \alpha y, w_{2}=x \beta z$ for $x, y, z \in(\Gamma \cup\{0,1\})^{*}$ and $\alpha, \beta \in \Gamma \cup\{0,1\}$ such that $\alpha<\beta$. In this case, for all $u \in D$, we have

$$
w_{1}<_{\operatorname{lex}} w_{1} 1 u<_{\operatorname{lex}} w_{2} \quad \text { and } \quad w_{1} 1 u \in \sigma(D) .
$$

Case 2. $w_{2}=w_{1} x$ for some $x \in(\Gamma \cup\{0,1\})^{+}$. Since $w_{2} \in \sigma(D)$, we have $x \notin 0^{*}$. Say $x=0^{j} \alpha y$ for some $j \geq 0, \alpha \neq 0$ and $y \in(\Gamma \cup\{0,1\})^{*}$. We must have $\alpha \in\left\{1, a, b_{1}, b_{2}, b_{3}, \sharp\right\}$. Since every symbol from this set is larger than 0 (see (16) ) we must have $\alpha>0$. Then for all $u \in D$, we have

$$
w_{1}<_{\operatorname{lex}} w_{1} 0^{j+1} 1 u<_{\operatorname{lex}} w_{2} \text { and } w_{1} 0^{j+1} 1 u \in \sigma(D) .
$$

Hence $\left(\sigma(D) ; \leq_{\text {lex }}\right)$ is indeed densely colored by $c$. This proves Claim 2 .

Since $\$$ is the minimum in the order $<$ on $\Sigma_{i}$, for any $u \in E, v, v^{\prime} \in \sigma(D)$ and $w, w^{\prime} \in F$, we have

$$
v<_{\operatorname{lex}} v^{\prime} \Longrightarrow u \$ v \$ w<_{\operatorname{lex}} u \$ v^{\prime} \$ w^{\prime} .
$$

Therefore,

$$
\begin{aligned}
\left(\pi^{-1}(u \$ \sigma(D) \$ F) \cap L\left(\operatorname{Run}_{\sigma(\mathcal{A}, E)}\right) ; \sqsubseteq\right) & \cong \sum_{v \in \sigma(D)}\left(\pi^{-1}(u \$ v \$ F) \cap L\left(\operatorname{Run}_{\sigma(\mathcal{A}, E)}\right) ; \sqsubseteq\right) \\
& \stackrel{\text { Claim } 1}{\cong} \sum_{v \in \sigma(D)}\left(\pi^{-1}(u c(v) \$ F) \cap L\left(\operatorname{Run}_{\mathcal{A}}\right) ; \sqsubseteq\right) \\
& \stackrel{\text { Claim } 2}{\cong} \operatorname{Shuf}\left(\left\{\left(\pi^{-1}(u v \$ F) \cap L\left(\operatorname{Run}_{\mathcal{A}}\right) ; \sqsubseteq\right) \mid v \in D\right\}\right) .
\end{aligned}
$$

6.2.2 Base case: automatic presentations for $L_{\bar{c}}^{1}, K^{1}$, and $M_{m}^{1}$ Recall the notations from Section 6.1.1. In the following, if $D$ is a regular language and $\mathcal{A}$ is a finite non-deterministic automaton then we denote by $D \mathcal{A}$ a finite automaton that results from the disjoint union of a deterministic automaton $\mathcal{A}_{D}$ for $D$ and the automaton $\mathcal{A}$ by adding all transitions $(q, a, p)$ where: (i) $q$ is a state of $\mathcal{A}_{D}$, (ii) there is a transition $\left(q, a, q^{\prime}\right)$ in $\mathcal{A}_{D}$, where $q^{\prime}$ is a final state of $\mathcal{A}_{D}$, and (iii) $p$ is an initial state of $\mathcal{A}$. Clearly, $L(D \mathcal{A})=D L(\mathcal{A})$. We will only apply this definition in case the product $D L(A)$ is unambiguous. This means that if $u \in D L(A)$ then there exists a unique factorization $u=u_{1} u_{2}$ with $u_{1} \in D$ and $u_{2} \in L(A)$. The following lemma is easy to prove:

Lemma 27. Let $\mathcal{A}$ be a finite non-deterministic automaton and let $D$ be a regular language such that the product $D L(A)$ is unambiguous. Let $u_{1} \in D$ and $u_{2} \in L(\mathcal{A})$. Then, the number of accepting runs of $D \mathcal{A}$ on $u_{1} u_{2}$ equals the number of accepting runs of $\mathcal{A}$ on $u_{2}$.

Lemma 28. From two given polynomials $q_{1}(\bar{x}), q_{2}(\bar{x}) \in \mathbb{N}[\bar{x}]$ in $k$ variables, one can effectively construct an automaton $\mathcal{A}\left[q_{1}, q_{2}\right]$ over the alphabet $\{a, \#, \$\}$ such that 
- $L\left(\mathcal{A}\left[q_{1}, q_{2}\right]\right)=\left(a^{+} \sharp\right)^{k} \$$ and

- For all $\bar{c} \in \mathbb{N}_{+}^{k},\left(\pi^{-1}\left(a^{\bar{c}} \$\right) \cap L\left(\operatorname{Run}_{\mathcal{A}\left[q_{1}, q_{2}\right]}\right) ; \sqsubseteq\right) \cong L\left[q_{1}(\bar{c}), q_{2}(\bar{c})\right]$.

Proof. We construct $\mathcal{A}\left[q_{1}, q_{2}\right]$ by taking a copy of $\mathcal{A}\left[C\left(q_{1}(\bar{x}), q_{2}(\bar{x})\right)\right]$ (see Lemma 24), adding a new state $q_{\$}$ and transitions $\left(q_{f}, \$, q_{\$}\right)$ for each accepting state $q_{f}$ in $\mathcal{A}\left[C\left(q_{1}(\bar{x}), q_{2}(\bar{x})\right)\right]$ and making $q_{\$}$ the only accepting state of $\mathcal{A}\left[q_{1}, q_{2}\right]$. Note that for any $\bar{c} \in \mathbb{N}_{+}^{k}$, the number of accepting runs of $\mathcal{A}\left[q_{1}, q_{2}\right]$ on $a^{\bar{c}} \$$ is the same as the number of accepting runs of $\mathcal{A}\left[C\left(q_{1}(\bar{x}), q_{2}(\bar{x})\right)\right]$ on $a^{\bar{c}}$, which is equal to $C\left(q_{1}(\bar{c}), q_{2}(\bar{c})\right)$. Hence, $\left(\pi^{-1}\left(a^{\bar{c}} \$\right) \cap L\left(\operatorname{Run}_{\mathcal{A}\left[q_{1}, q_{2}\right]}\right)\right.$; $\left.\sqsubseteq\right)$ forms a copy of $L\left[q_{1}(\bar{c}), q_{2}(\bar{c})\right]$ and the lemma is proved.

By Lemma 28, we can construct automata $\mathcal{A}_{1}=\mathcal{A}\left[p_{1}(\bar{x})+x_{\ell+1}, p_{2}(\bar{x})+x_{\ell+1}\right]$, where $\bar{x} \in \mathbb{N}_{+}^{\ell}$, over the alphabet $\{a, \sharp, \$\}, \mathcal{A}_{2}=\mathcal{A}\left[x_{1}+x_{2}, x_{1}+x_{2}\right]$ over the alphabet $\left\{b_{1}, \sharp, \$\right\}, \mathcal{A}_{3}=\mathcal{A}\left[x_{1}+x_{2}, x_{1}\right]$ over the alphabet $\left\{b_{2}, \sharp, \$\right\}$ and $\mathcal{A}_{4}=\mathcal{A}\left[x_{1}, x_{1}+x_{2}\right]$ over the alphabet $\left\{b_{3}, \sharp, \$\right\}$ such that:

$$
\begin{aligned}
\forall \bar{c} \in \mathbb{N}_{+}^{\ell} \forall c_{\ell+1} \in \mathbb{N}_{+}:\left(\pi^{-1}\left(a^{\bar{c} c_{\ell+1}} \$\right) \cap L\left(\operatorname{Run}_{\mathcal{A}_{1}}\right) ; \sqsubseteq\right) \cong L\left[p_{1}(\bar{c})+c_{\ell+1}, p_{2}(\bar{c})+c_{\ell+1}\right] \\
\forall e_{1}, e_{2} \in \mathbb{N}_{+}:\left(\pi^{-1}\left(b_{1}^{e_{1}} \sharp b_{1}^{e_{2}} \sharp \$\right) \cap L\left(\operatorname{Run}_{\mathcal{A}_{2}}\right) ; \sqsubseteq\right) \cong L\left[e_{1}+e_{2}, e_{1}+e_{2}\right] \\
\forall e_{1}, e_{2} \in \mathbb{N}_{+}:\left(\pi^{-1}\left(b_{2}^{e_{1}} \sharp b_{2}^{e_{2}} \sharp \$\right) \cap L\left(\operatorname{Run}_{\mathcal{A}_{3}}\right) ; \sqsubseteq\right) \cong L\left[e_{1}+e_{2}, e_{1}\right] \\
\forall e_{1}, e_{2} \in \mathbb{N}_{+}:\left(\pi^{-1}\left(b_{3}^{e_{1}} \sharp b_{3}^{e_{2}} \sharp \$\right) \cap L\left(\operatorname{Run}_{\mathcal{A}_{4}}\right) ; \sqsubseteq\right) \cong L\left[e_{1}, e_{1}+e_{2}\right]
\end{aligned}
$$

Define the following automata:

$$
\mathcal{A}_{1}^{0}=\mathcal{A}_{1} \uplus\left(\left(a^{+} \sharp\right)^{n}\left(\mathcal{A}_{3} \uplus \mathcal{A}_{4}\right)\right), \quad \mathcal{A}_{2}^{0}=\mathcal{A}_{2} \uplus\left(b_{1}^{+} \sharp\left(\mathcal{A}_{3} \uplus \mathcal{A}_{4}\right)\right), \quad \mathcal{A}_{3}^{0}=b_{2} \sharp\left(\mathcal{A}_{3} \uplus \mathcal{A}_{4}\right) .
$$

Note that

$$
\begin{aligned}
& L\left(\mathcal{A}_{1}^{0}\right)=\left(a^{+} \sharp\right)^{n}\left(\left(a^{+} \sharp\right)^{\ell-n+1} \cup\left(b_{2}^{+} \sharp\right)^{2} \cup\left(b_{3}^{+} \sharp\right)^{2}\right) \$, \\
& L\left(\mathcal{A}_{2}^{0}\right)=b_{1}^{+} \sharp\left(b_{1}^{+} \sharp \cup\left(b_{2}^{+} \sharp\right)^{2} \cup\left(b_{3}^{+} \sharp\right)^{2}\right) \$, \\
& L\left(\mathcal{A}_{3}^{0}\right)=b_{2} \sharp\left(\left(b_{2}^{+} \sharp\right)^{2} \cup\left(b_{3}^{+} \sharp\right)^{2}\right) \$ .
\end{aligned}
$$

Hence, applying Lemma 26 (with $F=\{\varepsilon\}$ ), we can effectively construct automata $\mathcal{A}_{j}^{1}(j \in$ $\{1,2,3\})$ as follows:

$$
\mathcal{A}_{1}^{1}=\sigma\left(\mathcal{A}_{1}^{0},\left(a^{+} \sharp\right)^{n}\right), \quad \mathcal{A}_{2}^{1}=\sigma\left(\mathcal{A}_{2}^{0}, b_{1}^{+} \sharp\right), \quad \mathcal{A}_{3}^{1}=\sigma\left(\mathcal{A}_{3}^{0}, b_{2} \sharp\right) .
$$

For all $\bar{c} \in \mathbb{N}_{+}^{n}$ we get:

$$
\begin{aligned}
& \left(\pi^{-1}\left(L\left(\mathcal{A}_{1}^{1}\right)\left[a^{\bar{c}}\right]\right) \cap L\left(\operatorname{Run}_{\mathcal{A}_{1}^{1}}\right) ; \sqsubseteq\right) \stackrel{\text { Lemma }}{\cong} \\
& \operatorname{Shuf}\left(\left\{\left(\pi^{-1}\left(a^{\bar{c}} v \$\right) \cap L\left(\operatorname{Run}_{\mathcal{A}_{1}^{0}}\right) ; \sqsubseteq\right) \mid v \in\left(a^{+} \sharp\right)^{\ell-n+1} \cup\left(b_{2}^{+} \sharp\right)^{2} \cup\left(b_{3}^{+} \sharp\right)^{2}\right\}\right)= \\
& \operatorname{Shuf}\left(\left\{\left(\pi^{-1}\left(a^{\bar{c} \bar{e}} \$\right) \cap L\left(\operatorname{Run}_{\mathcal{A}_{1}^{0}}\right) ; \sqsubseteq\right) \mid \bar{e} \in \mathbb{N}_{+}^{\ell-n+1}\right\} \cup\right. \\
& \left\{\left(\pi^{-1}\left(a^{\bar{c}} b_{2}^{e_{1}} \sharp b_{2}^{e_{2}} \sharp \$\right) \cap L\left(\operatorname{Run}_{\mathcal{A}_{1}^{0}}\right) ; \sqsubseteq\right) \mid e_{1}, e_{2} \in \mathbb{N}_{+}\right\} \cup \\
& \left.\left\{\left(\pi^{-1}\left(a^{\bar{c}} b_{3}^{e_{1}} \sharp b_{3}^{e_{2}} \sharp \$\right) \cap L\left(\operatorname{Run}_{\mathcal{A}_{1}^{0}}\right) ; \sqsubseteq\right) \mid e_{1}, e_{2} \in \mathbb{N}_{+}\right\}\right) \stackrel{\text { Lemma } 27}{\cong} \\
& \operatorname{Shuf}\left(\left\{\left(\pi^{-1}\left(a^{\bar{c} \bar{e}} \$\right) \cap L\left(\operatorname{Run}_{\mathcal{A}_{1}}\right) ; \sqsubseteq\right) \mid \bar{e} \in \mathbb{N}_{+}^{\ell-n+1}\right\} \cup\right. \\
& \left\{\left(\pi^{-1}\left(b_{2}^{e_{1}} \sharp b_{2}^{e_{2}} \sharp \$\right) \cap L\left(\operatorname{Run}_{\mathcal{A}_{3}}\right) ; \sqsubseteq\right) \mid e_{1}, e_{2} \in \mathbb{N}_{+}\right\} \cup \\
& \left.\left\{\left(\pi^{-1}\left(b_{3}^{e_{1}} \sharp b_{3}^{e_{2}} \sharp \$\right) \cap L\left(\operatorname{Run}_{\mathcal{A}_{4}}\right) ; \sqsubseteq\right) \mid e_{1}, e_{2} \in \mathbb{N}_{+}\right\}\right) \stackrel{(20)-(23)}{=} \\
& \operatorname{Shuf}\left(\left\{L\left[p_{1}(\bar{c}, \bar{e})+e_{\ell+1}, p_{2}(\bar{c}, \bar{e})+e_{\ell+1}\right] \mid \bar{e} \in \mathbb{N}_{+}^{\ell-n}, e_{\ell+1} \in \mathbb{N}_{+}\right\} \cup\right. \\
& \left.\left\{L\left[e_{1}+e_{2}, e_{1}\right] \mid e_{1}, e_{2} \in \mathbb{N}_{+}\right\} \cup\left\{L\left[e_{1}, e_{1}+e_{2}\right] \mid e_{1}, e_{2} \in \mathbb{N}_{+}\right\}\right) \stackrel{(99-[12]}{=} \\
& \operatorname{Shuf}\left(\mathcal{L}_{1}^{1}(\bar{c}) \cup \mathcal{L}_{3}^{1} \cup \mathcal{L}_{4}^{1}\right) \cong L_{\bar{c}}^{1}
\end{aligned}
$$


Similar calculations yield:

$$
\begin{aligned}
\forall m \in \mathbb{N}_{+}:\left(\pi^{-1}\left(L\left(\mathcal{A}_{2}^{1}\right)\left[b_{1}^{m} \sharp\right]\right)\right. & \left.\cap L\left(\operatorname{Run}_{\mathcal{A}_{2}^{1}}\right) ; \sqsubseteq\right) \\
\left(\pi^{-1}\left(L\left(\mathcal{A}_{3}^{1}\right)\left[b_{2} \sharp\right]\right) \cap L\left(\operatorname{Run}_{\mathcal{A}_{3}^{1}}\right) ; \sqsubseteq\right) & \left.\cong \operatorname{Shuf}\left(\mathcal{L}_{2}^{1}(m) \cup \mathcal{L}_{3}^{1} \cup \mathcal{L}_{4}^{1}\right) \cong \mathcal{L}_{3}^{1} \cup \mathcal{L}_{4}^{1}\right) \cong K^{1}
\end{aligned}
$$

Let $\mathcal{A}^{1}=\mathcal{A}_{1}^{1} \uplus \mathcal{A}_{2}^{1} \uplus \mathcal{A}_{3}^{1}$. It is easy to see that $L\left(\mathcal{A}^{1}\right)=\left(\left(a^{+} \sharp\right)^{n} \cup b_{1}^{+} \sharp \cup b_{2} \sharp\right) \$ R$ for some regular language $R \subseteq \Sigma_{1}^{+}$with first $(R) \subseteq\{0,1\}$. Hence $\mathcal{A}^{1}$ satisfies the statement in Proposition 25,

6.2.3 First inductive step: automatic presentations for $L_{\bar{c}}^{i+1}, K^{i+1}, M^{i+1}$ for $i$ odd Let $i \geq 1$ be an odd number. Recall the notations from Section 6.1.2. We write $k$ for $n-i$. By applying the inductive assumption, we obtain an automaton $\mathcal{A}^{i}$ such that $L\left(\mathcal{A}^{i}\right)=\left(\left(a^{+} \sharp\right)^{k+1} \cup\right.$ $\left.\beta \sharp \cup b_{2} \sharp\right) \$ R$ for some regular language $R \subseteq \Sigma_{i}^{*}$ where $\beta=b_{1}^{+}$if $i=1$, and $\beta=b_{1}$ otherwise. Furthermore, first $(R) \subseteq\{0,1\}$ and the following hold for $\mathcal{A}^{i}$ :

$$
\begin{aligned}
\forall \bar{c} \in \mathbb{N}_{+}^{k+1}: L_{\bar{c}}^{i} \cong\left(\pi^{-1}\left(a^{\bar{c}} \$ R\right) \cap L\left(\operatorname{Run}_{\mathcal{A}^{i}}\right) ; \sqsubseteq\right) \\
\mathcal{M}^{i} \cong\left\{\left(\pi^{-1}(u \sharp \$ R) \cap L\left(\operatorname{Run}_{\mathcal{A}^{i}}\right) ; \sqsubseteq\right) \mid u \in \beta\right\} \\
K^{i} \cong\left(\pi^{-1}\left(b_{2} \sharp \$ R\right) \cap L\left(\operatorname{Run}_{\mathcal{A}^{i}}\right) ; \sqsubseteq\right)
\end{aligned}
$$

For any $1 \leq j \leq n$, let $S_{j}=\$_{1}^{+} \cup \cdots \cup \$_{j}^{+}$. It is easy to see that

$$
\left(S_{j} ; \leq_{\text {lex }}\right) \cong \omega \cdot \mathbf{j}
$$

Define the automata $\mathcal{B}_{1}^{i}, \mathcal{B}_{2}^{i}$, and $\mathcal{B}_{3}^{i}$ as

$$
\begin{aligned}
& \mathcal{B}_{1}^{i}=\left(\left(a^{+} \sharp\right)^{k+1} \$ R \cap \mathcal{A}^{i}\right) \uplus\left(a^{+} \sharp\right)^{k+1} \$ S_{i}, \\
& \mathcal{B}_{2}^{i}=\left(\beta \sharp \$ R \cap \mathcal{A}^{i}\right) \uplus \beta \sharp \$ S_{i}, \\
& \mathcal{B}_{3}^{i}=\left(b_{2} \sharp \$ R \cap \mathcal{A}^{i}\right) \uplus b_{2} \sharp \$ S_{i} .
\end{aligned}
$$

By (16), (24)-(27), and the fact that first $(R) \subseteq\{0,1\}$, we have

$$
\begin{aligned}
& \forall \bar{c} \in \mathbb{N}_{+}^{k+1}:\left(\pi^{-1}\left(a^{\bar{c}} \$\left(S_{i} \cup R\right)\right) \cap L\left(\operatorname{Run}_{\mathcal{B}_{1}^{i}}\right) ; \sqsubseteq\right) \cong \omega \cdot \mathbf{i}+L_{\bar{c}}^{i}, \\
&\left\{\left(\pi^{-1}\left(u \sharp \$\left(S_{i} \cup R\right)\right) \cap L\left(\operatorname{Run}_{\mathcal{B}_{2}^{i}}\right) ; \sqsubseteq\right) \mid u \in \beta\right\} \cong\left\{\omega \cdot \mathbf{i}+M \mid M \in \mathcal{M}^{i}\right\}, \\
&\left(\pi^{-1}\left(b_{2} \sharp \$\left(S_{i} \cup R\right)\right) \cap L\left(\operatorname{Run}_{\mathcal{B}_{3}^{i}}\right) ; \sqsubseteq\right) \cong \omega \cdot \mathbf{i}+K^{i} .
\end{aligned}
$$

Now construct the automata $\mathcal{C}_{1}^{i}, \mathcal{C}_{2}^{i}$, and $\mathcal{C}_{3}^{i}$ as follows:

$$
\mathcal{C}_{1}^{i}=\mathcal{B}_{1}^{i} \uplus\left(a^{+} \sharp\right)^{k} \mathcal{B}_{2}^{i}, \quad \mathcal{C}_{2}^{i}=b_{1} \sharp \mathcal{B}_{2}^{i}, \quad \mathcal{C}_{3}^{i}=b_{2} \sharp\left(\mathcal{B}_{2}^{i} \uplus \mathcal{B}_{3}^{i}\right) .
$$

We have

$$
\begin{aligned}
& L\left(\mathcal{C}_{1}^{i}\right)=\left(a^{+} \sharp\right)^{k}\left(a^{+} \sharp \cup \beta \sharp\right) \$\left(S_{i} \cup R\right), \\
& L\left(\mathcal{C}_{2}^{i}\right)=b_{1} \sharp \beta \sharp \$\left(S_{i} \cup R\right), \\
& L\left(\mathcal{C}_{3}^{i}\right)=b_{2} \sharp\left(\beta \sharp \cup b_{2} \sharp\right) \$\left(S_{i} \cup R\right) .
\end{aligned}
$$

Hence, we can apply Lemma 26 to $\mathcal{C}_{1}^{i}, \mathcal{C}_{2}^{i}$, and $\mathcal{C}_{3}^{i}$ (with $F=S_{i} \cup R$ ) to define the following automata:

$$
\mathcal{A}_{1}^{i+1}=\sigma\left(\mathcal{C}_{1}^{i},\left(a^{+} \sharp\right)^{k}\right), \quad \mathcal{A}_{2}^{i+1}=\sigma\left(\mathcal{C}_{2}^{i}, b_{1} \sharp\right), \quad \mathcal{A}_{3}^{i+1}=\sigma\left(\mathcal{C}_{3}^{i}, b_{2} \sharp\right) .
$$


For all $\bar{c} \in \mathbb{N}_{+}^{k}$ we get:

$$
\begin{gathered}
\left(\pi^{-1}\left(L\left(\mathcal{A}_{1}^{i+1}\right)\left[a^{\bar{c}}\right]\right) \cap L\left(\operatorname{Run}_{\mathcal{A}_{1}^{i+1}}\right) ; \sqsubseteq\right) \stackrel{\operatorname{Lemma}}{\cong} \underline{26} \\
\operatorname{Shuf}\left(\left\{\left(\pi^{-1}\left(a^{\bar{c}} v \$\left(S_{i} \cup R\right)\right) \cap L\left(\operatorname{Run}_{\mathcal{C}_{1}^{i}}\right) ; \sqsubseteq\right) \mid v \in a^{+} \sharp \cup \beta \sharp\right\}\right)= \\
\operatorname{Shuf}\left(\left\{\left(\pi^{-1}\left(a^{\bar{c} e} \$\left(S_{i} \cup R\right)\right) \cap L\left(\operatorname{Run}_{\mathcal{C}_{1}^{i}}\right) ; \sqsubseteq\right) \mid e \in \mathbb{N}_{+}\right\} \cup\right. \\
\left.\left\{\left(\pi^{-1}\left(a^{\bar{c}} u \sharp \$\left(S_{i} \cup R\right)\right) \cap L\left(\operatorname{Run}_{\mathcal{C}_{1}^{i}}\right) ; \sqsubseteq\right) \mid u \in \beta\right\}\right) \stackrel{\text { Lemma } 27}{\cong} \\
\operatorname{Shuf}\left(\left\{\left(\pi^{-1}\left(a^{\bar{c} e} \$\left(S_{i} \cup R\right)\right) \cap L\left(\operatorname{Run}_{\mathcal{B}_{1}^{i}}\right) ; \sqsubseteq\right) \mid e \in \mathbb{N}_{+}\right\} \cup\right. \\
\left.\left\{\left(\pi^{-1}\left(u \sharp \$\left(S_{i} \cup R\right)\right) \cap L\left(\operatorname{Run}_{\mathcal{B}_{2}^{i}}\right) ; \sqsubseteq\right) \mid u \in \beta\right\}\right) \stackrel{\text { [1], (32) }}{=} \\
\operatorname{Shuf}\left(\left\{\omega \cdot \mathbf{i}+\mathcal{L}_{\bar{c} e}^{i} \mid e \in \mathbb{N}_{+}\right\} \cup\left\{\omega \cdot \mathbf{i}+M \mid M \in \mathcal{M}^{i}\right\}\right) \stackrel{\text { (13), (14) }}{\cong} L_{\bar{c}}^{i+1}
\end{gathered}
$$

Similarly, we can show:

$$
\begin{aligned}
& \left(\pi^{-1}\left(L\left(\mathcal{A}_{2}^{i+1}\right)\left[b_{1} \sharp\right]\right) \cap L\left(\operatorname{Run}_{\mathcal{A}_{2}^{i+1}}\right) ; \sqsubseteq\right) \cong \operatorname{Shuf}\left(\left\{\omega \cdot \mathbf{i}+M \mid M \in \mathcal{M}^{i}\right\}\right) \cong M^{i+1}, \\
& \left(\pi^{-1}\left(L\left(\mathcal{A}_{3}^{i+1}\right)\left[b_{2} \sharp\right]\right) \cap L\left(\operatorname{Run}_{\mathcal{A}_{3}^{i+1}}\right) ; \sqsubseteq\right) \cong \operatorname{Shuf}\left(\left\{\omega \cdot \mathbf{i}+M \mid M \in \mathcal{M}^{i}\right\} \cup\left\{\omega \cdot \mathbf{i}+K^{i}\right\}\right) \cong K^{i+1} .
\end{aligned}
$$

Let $\mathcal{A}^{i+1}=\mathcal{A}_{1}^{i+1} \uplus \mathcal{A}_{2}^{i+1} \uplus \mathcal{A}_{3}^{i+1}$. It is easy to see that $L\left(\mathcal{A}^{i+1}\right)=\left(\left(a^{+} \sharp\right)^{k} \cup b_{1} \sharp \cup b_{2} \sharp\right) \$ R^{\prime}$ for some regular language $R^{\prime} \subseteq \Sigma_{i+1}^{+}$with first $\left(R^{\prime}\right) \subseteq\{0,1\}$. Hence $\mathcal{A}^{i+1}$ satisfies the statement in Proposition 25.

6.2.4 Second inductive step: automatic presentations for $L_{\bar{c}}^{i+1}, K^{i+1}, M^{i+1}$ for $i$ even Using the same technique, we can construct automatic presentations for $L_{\bar{c}}^{i+1}\left(\bar{c} \in \mathbb{N}_{+}^{k}\right)$, $M^{i+1}$, and $K^{i+1}$ in case $i$ is even. We first define the automata $\mathcal{B}_{1}^{i}, \mathcal{B}_{2}^{i}$, and $\mathcal{B}_{3}^{i}$ as in (28)-(30), with $\beta=b_{1}$ this time. Then we construct

$$
\mathcal{C}_{1}^{i}=\mathcal{B}_{1}^{i} \uplus\left(a^{+} \sharp\right)^{k} \mathcal{B}_{3}^{i}, \quad \mathcal{C}_{2}^{i}=b_{1} \sharp\left(\mathcal{B}_{2}^{i} \uplus \mathcal{B}_{3}^{i}\right), \quad \mathcal{C}_{3}^{i}=b_{2} \sharp \mathcal{B}_{3}^{i} .
$$

We define the following automata by applying Lemma 26.

$$
\mathcal{A}_{1}^{i+1}=\sigma\left(\mathcal{C}_{1}^{i},\left(a^{+} \sharp\right)^{k}\right), \quad \mathcal{A}_{2}^{i+1}=\sigma\left(\mathcal{C}_{2}^{i}, b_{1} \sharp\right), \quad \mathcal{A}_{3}^{i+1}=\sigma\left(\mathcal{C}_{3}^{i}, b_{2} \sharp\right) .
$$

By Lemma 26, it is easy to check the following:

$$
\begin{aligned}
& \forall \bar{c} \in \mathbb{N}_{+}^{k}:\left(\pi^{-1}\left(L\left(\mathcal{A}_{1}^{i+1}\right)\left[a^{\bar{c}}\right]\right) \cap L\left(\operatorname{Run}_{\mathcal{A}_{1}^{i+1}}\right) ; \sqsubseteq\right) \cong \operatorname{Shuf}\left(\left\{\omega \cdot \mathbf{i}+\mathcal{L}_{\bar{c} x}^{i} \mid x \in \mathbb{N}_{+}\right\} \cup\left\{\omega \cdot \mathbf{i}+K^{i}\right\}\right) \\
& \cong L_{\bar{c}}^{i+1}, \\
&\left(\pi^{-1}\left(L\left(\mathcal{A}_{2}^{i+1}\right)\left[b_{1} \sharp\right]\right) \cap L\left(\operatorname{Run}_{\mathcal{A}_{2}^{i+1}}\right) ; \sqsubseteq\right) \cong \operatorname{Shuf}\left(\left\{\omega \cdot \mathbf{i}+M^{i}\right\} \cup\left\{\omega \cdot \mathbf{i}+K^{i}\right\}\right) \\
& \cong M^{i+1}, \\
&\left(\pi ^ { - 1 } ( L ( \mathcal { A } _ { 3 } ^ { i + 1 } ) [ b _ { 2 } \sharp ] ) \cap L \left(\operatorname{Run}_{\left.\left.\mathcal{A}_{3}^{i+1}\right) ; \sqsubseteq\right)} \cong \operatorname{Shuf}\left(\left\{\omega \cdot \mathbf{i}+K^{i}\right\}\right)\right.\right. \\
& \\
& \cong K^{i+1} .
\end{aligned}
$$

Let $\mathcal{A}^{i+1}=\mathcal{A}_{1}^{i+1} \uplus \mathcal{A}_{2}^{i+1} \uplus \mathcal{A}_{3}^{i+1}$. It is easy to see that $L\left(\mathcal{A}^{i+1}\right) \subseteq\left(\left(a^{+} \sharp\right)^{k} \cup b_{1} \sharp \cup b_{2} \sharp\right) \$ R^{\prime}$ for some regular language $R^{\prime} \subseteq \Sigma_{i+1}^{+}$with first $\left(R^{\prime}\right) \subseteq\{0,1\}$. Hence $\mathcal{A}^{i+1}$ satisfies the statement in Proposition 25. This finishes the construction in the inductive step and hence the proof of Proposition 25, Hence we obtain:

Theorem 29. The isomorphism problem for the class of automatic linear orders is at least as hard as $\operatorname{FOTh}(\mathbb{N} ;+, \times)$. 
In [15, it is shown that every linear order has finite FC-rank. We do not define the FC-rank of a linear order in general, see e.g. [15]. A linear order $(L, \leq)$ has FC-rank 1, if after identifying all $x, y \in L$ such that the interval $[x, y]$ is finite, one obtains a dense ordering or the singleton linear order. The result of [15] mentioned above suggests that the isomorphism problem might be simpler for linear orders of low FC-rank. We now prove that this is not the case:

Corollary 30. The isomorphism problem for automatic linear orders of FC-rank 1 is at least as hard as $\operatorname{FOTh}(\mathbb{N} ;+, \times)$.

Proof. We provide a reduction from the isomorphism problem for automatic linear orders (of arbitrary rank): if $(L, \leq)$ is an automatic linear order, then so is $(K, \leq)=((-1,0]+[1,2)) \cdot(L, \leq)$ (this linear order is obtained from $L$ by replacing each point with a copy of the rational numbers in $(-1,0] \cup[1,2))$. Then $(K, \leq)$ has FC-rank 1: Only the copies of 0 and 1 will be identified, and the resulting order is isomorphic to $(\mathbb{Q}, \leq)$. Moreover, $(L, \leq)$ is isomorphic to the set of all $x \in K$ satisfying $\exists z>x \forall y:(x<y \leq z \rightarrow y=z)$. Hence $(L, \leq) \cong\left(L^{\prime}, \leq^{\prime}\right)$ if and only if $((-1,0]+[1,2)) \cdot(L, \leq) \cong((-1,0]+[1,2)) \cdot\left(L^{\prime}, \leq^{\prime}\right)$, which completes the reduction.

\section{Conclusion}

This paper looks at the isomorphism problem of some typical classes of automatic structures. Such classes include equivalence structures, successor trees of height at most $n \in \mathbb{N}$, and linear orders. In particular, we demonstrate, respectively, $\Pi_{1}^{0}$-completeness and $\Pi_{2 n-3}^{0}$-completeness for the isomorphism problem of the first two classes. The uniformity in our proof shows that the isomorphism problem of automatic trees of finite height is recursively equivalent to $\operatorname{FOTh}(\mathbb{N} ;+, \times)$. Similarly, we prove that the isomorphism problem of automatic linear orders is at least as hard as $\operatorname{FOTh}(\mathbb{N} ;+, \times)$. The same technique is also used to proved that the isomorphism problem of recursive trees of height at most $n$ is $\Pi_{2 n}^{0}$-complete.

We conclude with an application of Theorems 18 and 29. The following corollary shows that although automatic structures look simple (especially for automatic trees), there may be no "simple" isomorphism between two automatic copies of the same structure. An isomorphism $f$ between two automatic structures with domains $L_{1}$ and $L_{2}$, respectively, is a $\Sigma_{k}^{0}$-isomorphism, if the set $\left\{(x, f(x)) \mid x \in L_{1}\right\}$ belongs to $\Sigma_{k}^{0}$.

Corollary 31. For any $k \in \mathbb{N}$, there exist two isomorphic automatic trees of finite height (and two automatic linear orders) without any $\Sigma_{k}^{0}$-isomorphism.

Proof. Let $T_{1}=\left(D_{1} ; E_{1}\right)$ and $T_{2}=\left(D_{2} ; E_{2}\right)$ be two automatic trees. Let $P_{1}(x, y), P_{2}(x, y), \ldots$ be an effective enumeration of all binary $\Sigma_{k}^{0}$-predicates. This means that from given $e \geq 1$ we can effectively compute a description (e.g. a $\Sigma_{k}$-formula over $\left.(\mathbb{N} ;+, \times)\right)$ of the predicate $P_{e}(x, y)$. We define the statement iso $\left(T_{1}, T_{2}, k\right)$ as follows:

$$
\begin{aligned}
& \exists e \forall x_{1}, x_{2} \in D_{1} \exists y_{1}, y_{2} \in D_{2}: \begin{array}{l}
P_{e}\left(x_{1}, y_{1}\right) \wedge P_{e}\left(x_{2}, y_{2}\right) \wedge \\
\end{array} \\
&\left.\wedge \forall y \in D_{2} \exists x \in D_{1}: x_{2} \leftrightarrow y_{1}=y_{2}\right) \wedge\left(\left(x_{1}, x_{2}\right) \in E_{1} \leftrightarrow\left(y_{1}, y_{2}\right) \in E_{2}\right)
\end{aligned}
$$

Since $P_{e}$ is a $\Sigma_{k}^{0}$-predicate, this is a $\Sigma_{k+2}^{0}$-statement, which expresses the existence of a $\Sigma_{k^{-}}^{0}$ isomorphism from $T_{1}$ to $T_{2}$.

By Theorem 18, there is a natural number $n$ such that the isomorphism problem on the class $\mathcal{T}_{n}$ of automatic trees of height at most $n$ is $\Sigma_{k+3}$-hard. If for all $T_{1}, T_{2} \in \mathcal{T}_{n}$ with $T_{1} \cong T_{2}$ there exists a $\Sigma_{k}^{0}$-isomorphism from $T_{1}$ to $T_{2}$, then the isomorphism problem on $\mathcal{T}_{n}$ reduces to checking existence of a $\Sigma_{k}^{0}$-isomorphism, which is in $\Sigma_{k+2}^{0}$ by the above consideration. Hence, there must be $T_{1}, T_{2} \in \mathcal{T}_{n}$ with $T_{1} \cong T_{2}$ but there is no $\Sigma_{k}^{0}$-isomorphism between them.

The corollary for linear orders can be proved in the same way, where in the definition of iso $\left(T_{1}, T_{2}, k\right)$ we replace $\left(x_{1}, x_{2}\right) \in E_{1} \leftrightarrow\left(y_{1}, y_{2}\right) \in E_{2}$ with $x_{1}<_{1} x_{2} \leftrightarrow y_{1}<_{2} y_{2}$, where $<_{1}$ and $<_{2}$ are the linear orders of $T_{1}$ and $T_{2}$, respectively. 


\section{References}

1. V. Bárány, L. Kaiser, and S. Rubin. Cardinality and counting quantifiers on omega-automatic structures. In Proceedings of STACS 2008, pages 385-396. IFIB Schloss Dagstuhl, 2008.

2. A. Blumensath and E. Grädel. Automatic structures. In Proceedings of LICS 2000, pages 51-62. IEEE Computer Society Press, 2000.

3. A. Blumensath and E. Grädel. Finite presentations of infinite structures: Automata and interpretations. Theory Comput. Syst., 37(6):641-674, 2004.

4. W. Calvert and J. F. Knight. Classification from a computable viewpoint. Bull. Symbolic Logic, 12(2):191-218, 2006.

5. C. Elgot. Decision problems of finite automata design and related arithmetics. Trans. Am. Math. Soc., 98:21-51, 1961 .

6. D. B. A. Epstein, J. W. Cannon, D. F. Holt, S. V. F. Levy, M. S. Paterson, and W. P. Thurston. Word processing in groups. Jones and Bartlett, Boston, 1992.

7. S. S. Goncharov and J. F. Knight. Computable structure and antistructure theorems. Algebra i Logika, 41(6):639-681, 2002.

8. B. R. Hodgson. On direct products of automaton decidable theories. Theoret. Comput. Sci., 19:331335, 1982.

9. J. Honkala. On the problem whether the image of an $N$-rational series equals $N$. Fund. Inform., 73(1-2):127-132, 2006.

10. J. E. Hopcroft and J. D. Ullman. Introduction to automata theory, languages and computation. Addison-Wesley, Reading, MA, 1979.

11. H. Ishihara, B. Khoussainov, and S. Rubin. Some results on automatic structures. In Proceedings of LICS 2002, pages 235-244. IEEE Computer Society Press, 2002.

12. B. Khoussainov and M. Minnes. Model theoretic complexity of automatic structures. In Proceedings of TAMC 2008, number 4978 in Lecture Notes in Computer Science, pages 514-525. Springer, 2008.

13. B. Khoussainov and A. Nerode. Automatic presentations of structures. In LCC: International Workshop on Logic and Computational Complexity, number 960 in Lecture Notes in Computer Science, pages 367-392, 1995.

14. B. Khoussainov, A. Nies, S. Rubin, and F. Stephan. Automatic structures: richness and limitations. Log. Methods Comput. Sci., 3(2):2:2, 18 pp. (electronic), 2007.

15. B. Khoussainov, S. Rubin, and F. Stephan. Automatic linear orders and trees. ACM Trans. Comput. Log., 6(4):675-700, 2005.

16. D. Kuske and M. Lohrey. Some natural decision problems in automatic graphs. J. Symbolic Logic, 2009. to appear.

17. Y. V. Matiyasevich. Hilbert's Tenth Problem. MIT Press, Cambridge, Massachusetts, 1993.

18. A. Nies. Describing groups. Bull. Symbolic Logic, 13(3):305-339, 2007.

19. H. Rogers. Theory of Recursive Functions and Effective Computability. McGraw-Hill, 1968.

20. J. Rosenstein. Linear Ordering. Academic Press, 1982.

21. S. Rubin. Automatic Structures. PhD thesis, University of Auckland, 2004.

22. S. Rubin. Automata presenting structures: A survey of the finite string case. Bull. Symbolic Logic, 14:169-209, 2008.

23. R. I. Soare. Recursively enumerable sets and degrees. Perspectives in Mathematical Logic. Springer, 1987.

24. T. Tsankov. The additive group of the rationals does not have an automatic presentation. http://arxiv.org/abs/0905.1505 